\title{
Wallcrossing and cohomology of the moduli space of Hitchin pairs
}

Wu-yen Chuang, Duiliu-Emanuel Diaconescu and Guang Pan

A conjectural recursive relation for the Poincaré polynomial of the Hitchin moduli space is derived from wallcrossing in the refined local Donaldson-Thomas theory of a curve. A doubly refined generalization of this theory is also conjectured and shown to similarly determine the Hodge polynomial of the same moduli space.

1. Introduction

1.1. Refined wallcrossing conjectures

1.2. Recursion formula for refined Higgs invariants

1.3. Higgs invariants and cohomology of moduli spaces of Hitchin pairs

1.4. Asymptotic refined ADHM invariants

2. ADHM invariants, Hitchin pairs and wallcrossing

2.1. Review of ADHM sheaves

2.2. Connection with Hitchin pairs

2.3. Remarks on refined wallcrossing conjectures

2.4. Derivation of recursion formula

3. Asymptotic refined ADHM invariants from gauge theory 22

3.1. Geometric engineering via local ruled surfaces

3.2. Hirzebruch genus 
2 Wu-yen Chuang, Duiliu-Emanuel Diaconescu and Guang Pan

3.3. Comparison with the ruled vertex

3.4. Refinement

3.5. Double refinement

3.6. Localization interpretation for $r=2$

4. Examples, comparison with existing results

4.1. Rank $r=1$

4.2. Rank $r=2$

4.3. Rank $r=3$

Appendix A. Existing results

A.1. Rank $r=2$

A.2. Rank $r=3$

A.3.Hausel-Rodriguez-Villegas formula

\section{Introduction}

Let $X$ be a smooth projective curve over $\mathbb{C}$ and $M_{1}, M_{2}$ be line bundles on $X$ so that $M_{1} \otimes_{X} M_{2} \simeq K_{X}^{-1}$. Given any such triple $\mathcal{X}=\left(X, M_{1}, M_{2}\right)$, the total space $Y$ of the rank two bundle $M_{1}^{-1} \oplus M_{2}^{-1}$ is a quasi-projective three-fold with trivial canonical class. The stable pair theory [55] of such three-folds has been reformulated in [13] as an enumerative theory of twisted quiver 
sheaves on $X$. The later are twisted representations of a quiver of the form

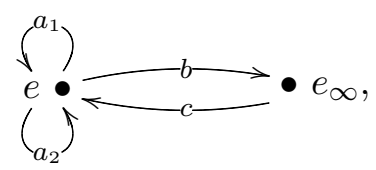

with a relation of the form $\left[a_{1}, a_{2}\right]+c b$, in the abelian category $\mathfrak{C o h}(X)$ of coherent sheaves on $X$. In more detail, one assigns coherent $\mathcal{O}_{X}$-modules $\left(E, E_{\infty}\right)$ to the nodes $\left(e, e_{\infty}\right)$, and twisted morphisms $\Phi_{i}: E \otimes_{X} M_{i} \rightarrow E$, $i=1.2, \phi: E \otimes_{X} \otimes_{X} M_{2} \rightarrow E_{\infty}, \psi: E_{\infty} \rightarrow E$ to the arrows $\left(a_{1}, a_{2}, b, c\right)$, satisfying the relation

$$
\Phi_{1} \circ\left(\Phi_{2} \otimes 1_{M_{1}}\right)-\Phi_{2} \circ\left(\Phi_{1} \otimes 1_{M_{2}}\right)+\psi \circ \phi=0 .
$$

The above quiver will be referred to as an ADHM quiver, and the resulting quiver sheaves as ADHM sheaves on $X$. Note that such objects form naturally an abelian category which contains in particular an abelian subcategory $\mathcal{C}_{\mathcal{X}}$ of quiver sheaves with $E_{\infty} \simeq V \otimes \mathcal{O}_{X}$, where $V$ is a finite-dimensional complex vector space. The numerical invariants of objects in $\mathcal{C}_{\mathcal{X}}$ will be denoted by $(r, e, v) \in \mathbb{Z}_{\geq 0} \times \mathbb{Z} \times \mathbb{Z}_{\geq 0}$ where $(r, e)$ are the rank and degree of $E$, respectively, and $v=\operatorname{dim}(V)$. For the purpose of the present paper, it is important to note that the $v=0$ objects of $\mathcal{C}_{\mathcal{X}}$ are Higgs sheaves on $X$ i.e., coherent $\mathcal{O}_{X}$-modules $E$ decorated by a morphism $E \otimes_{X}\left(M_{1} \oplus M_{2}\right) \rightarrow E$ satisfying the standard integrability condition

$$
\Phi_{1} \circ\left(\Phi_{2} \otimes 1_{M_{1}}\right)-\Phi_{2} \circ\left(\Phi_{1} \otimes 1_{M_{2}}\right)=0
$$

Obviously, such objects form an abelian subcategory of $\mathcal{C}_{\mathcal{X}}$.

As shown in $[7,8,12]$, there is a natural slope stability condition on $\mathcal{C}_{\mathcal{X}}$ parameterized by a real number $\delta \in \mathbb{R}$, which exhibits a nontrivial chamber structure. Moreover, the Joyce-Song theory of generalized DonaldsonThomas invariants [38] applies to ADHM sheaves, resulting in counting invariants defined via Behrend's constructible function [3]. The counting invariants of $v=1$ objects in the asymptotic chamber $\delta>>0$ were related to stable pair invariants of $Y$ in [13].

In string theory, generalized Donaldson-Thomas invariants of ADHM sheaves represent counting invariants of BPS bound states of D6-D2-D0 brane configurations on $Y$ with multiplicities $(v, r, e)$, respectively. Since $Y$ is noncompact, the gauge bundle on the D6-brane world volume is fixed to be trivial and treated as framing data. In particular, for $v=0$, the invariants in question count D2-D0 bound states. According to the strong rationality 
conjecture formulated in [55], asymptotic $v=1$ invariants are expected to be also related to Gopakumar-Vafa invariants of $Y$, that is numbers of BPS states of M-theory membranes wrapping holomorphic cycles in $Y$. The later are in turn related to Nekrasov instanton sums in five-dimensional supersymmetric gauge theory. As shown in detail later, this connection yields an explicit conjectural formula for the refined asymptotic $v=1$ theory, which plays an important role in the present work.

The purpose of this paper is to present an application of ADHM sheaves to computations of Betti and Hodge numbers of moduli spaces of stable Hitchin pairs on the curve $X$. As a brief history of the subject, note that the Poincaré polynomial of the moduli space of stable bundles on a curve has been recursively computed in $[11,26]$ using number theoretic methods, respectively, [2] using gauge theoretic methods. The Hodge polynomial of the same moduli spaces, has been recursively computed in [18], and also in $[9,46-48]$ for bundles of rank two and three. ${ }^{1}$ The Poincaré polynomial of the moduli space of stable Hitchin pairs with coprime rank and degree has been computed by Hitchin in [29] for rank two, and Gothen [24], for rank three. Using number theoretic techniques, a conjectural formula for any rank has been derived by Hausel and Rodriguez-Villegas in [28] and generalized to Hodge polynomials by Hausel in [27]. Similar results for parabolic rank three Higgs bundles have been obtained in [22]. Finally, the motive of the moduli space of rank four Hitchin pairs in the Grothedieck ring of algebraic varieties is computed in the upcoming work [1].

This paper presents a string theoretic perspective on this subject based on wallcrossing and refined generalized Donaldson-Thomas invariants associated to ADHM sheaves. There are currently two theories of DonaldsonThomas invariants, the Kontsevich-Soibelman theory [42] and the JoyceSong theory [38]. The former is based on a construction of motivic Donaldson-Thomas invariants which specialize to integral valued invariants in a semiclassical limit. The later constructs $\mathbb{Q}$-valued generalized Donaldson-Thomas invariants which are conjecturally related to these integral invariants by a multicover formula [38, Section 6.2]. The application presented below relies on the motivic Donaldson-Thomas theory of Kontsevich and Soibelman applied to ADHM sheaves, or, equivalently, on a conjectural refinement of Joyce-Song theory.

As mentioned above, generalized Donaldson-Thomas theory of ADHM sheaves has been studied using the formalism of [38] in $[7,8,12]$. The main

\footnotetext{
${ }^{1}$ According to [18], the Hodge polynomial of the moduli space of rank three bundles has been first derived by P. Newstead in unpublished work.
} 
results for $v=1$ objects, which is the relevant case in this paper, are reviewed in Section 2.1. In particular for fixed numerical invariants $\gamma=(r, e) \in \mathbb{Z}_{\geq 1} \times$ $\mathbb{Z}$ there is a finite set of critical stability parameters dividing the real axis into stability chambers. Note that $\delta=0$ is critical for all $(r, e) \in \mathbb{Z}_{\geq 1} \times \mathbb{Z}$. Residual ADHM invariants $A_{\delta}(r, e)$ are defined in each chamber by equivariant virtual integration [12]. Wallcrossing formulas for ADHM invariants are derived in [7] using the formalism of Joyce [34-37] and Joyce and Song [38]. The resulting wallcrossing formulas are also shown to be in agreement with the Kontsevich-Soibelman formula [42]. Note that the theory of Joyce and Song also yields residual generalized Donaldson-Thomas invariants $H(r, e)$ counting semistable Higgs sheaves on $X$ with numerical invariants $(r, e) \in \mathbb{Z}_{\geq 1} \times \mathbb{Z}$. These invariants enter the wallcrossing formulas for $A_{\delta}(r, e)$ derived in [7].

The conjectures formulated in Section 1.1 below summarize the main results of Kontsevich-Soibelman theory needed in this paper. Since the virtual enumerative theory of ADHM sheaves has been studied in [7,12] employing Joyce-Song theory, these conjectures can be also viewed as a refinement of their generalized Donaldson-Thomas formalism. In particular, the conjectural invariants take in general values in a field of rational functions in one or two formal variables and are conjecturally related to the quantum Donaldson-Thomas invariants of Kontsevich and Soibelman by a refined multicover formula.

In order to make contact with previous results, note that refined wallcrossing formulas have been derived in physical theories defined by a SeibergWitten curve in $[6,15-17,21]$, and conjectured to hold in more general situations. Moreover, motivic wallcrossing formulas for Donaldson-Thomas invariants of quivers with potential have been also announced in [41]. The wallcrossing formulas conjectured in this paper for refined generalized Donaldson-Thomas invariants, are related to those of $[6,16,17,21]$ by a refined multicover formula, as explained in more detail below. In addition, it is worth noting that the invariants conjectured here are also equivariant residual invariants with respect to a torus action. Therefore, a rigorous construction would require an equivariant localization theorem for refined Donaldson-Thomas invariants. Although the conjectures below are specifically formulated for ADHM sheaves, analogous conjectures can be formulated in more general situations including abelian categories of coherent sheaves or coherent perverse sheaves on Calabi-Yau three-folds. Previous results and conjectures in the mathematics literature are presented in $[4,49]$.

The main application of the conjectures in Section 1.1 is a recursive formula presented in Section 1.2. This formula determines the Poincaré and 
Hodge polynomial of moduli spaces of Hitchin pairs with coprime rank and degree in terms of asymptotic refined ADHM invariants. The later are in turn determined by string theoretic techniques, the results being summarized in Section 1.4. In Section 4 it is checked by direct computation that the resulting expressions are in agreement with the results of [24,27-29] in many concrete examples. This provides strong evidence for the validity of the conjectural formalism proposed here. Note that Higgs sheaves on curves are also employed in [23] as a computational device for local BPS invariants of toric surfaces.

\subsection{Refined wallcrossing conjectures}

In order to fix the notation, let $\Delta(r, e) \subset \mathbb{R}_{>0}$ be the (finite) set of positive critical stability parameters of type $(r, e) \in \mathbb{Z}_{\geq 1} \times \mathbb{Z}$. For any $n \in \mathbb{Z}$, and any formal variable $y$ let

$$
[n]_{y}=\frac{y^{n}-y^{-n}}{y-y^{-1}} \in \mathbb{Q}(y) .
$$

Conjecture 1.1. Let $\gamma=(r, e) \in \mathbb{Z}_{\geq 1} \times \mathbb{Z}$. Then there exist refined equivariant residual $A D H M$ invariants $A_{\delta}(r, e)(y) \in \mathbb{Q}(y)$, for any $\delta \in \mathbb{R}$, and refined equivariant residual Higgs sheaf invariants $H(r, e)(y) \in \mathbb{Q}(y)$ so that $A_{\delta}(r, e)(1)=A_{\delta}(r, e), H(r, e)(1)=H(r, e)$ and the following wallcrossing formulas hold:

(i) Let $\delta_{c} \in \Delta(r, e)$ be a critical stability parameter and $\delta_{c-}<\delta_{c}, \delta_{c+}>$ $\delta_{c}$ be noncritical stability parameters so that $\left[\delta_{c-}, \delta_{c}\right) \cap \Delta(r, e)=\emptyset,\left(\delta_{c}, \delta_{c+}\right] \cap$ $\Delta(r, e)=\emptyset$. The following wallcrossing formula holds for $\delta_{c \pm}$ sufficiently close to $\delta_{c}$ :

$$
\begin{aligned}
& A_{\delta_{c}+}(\gamma)(y)-A_{\delta_{c}-}(\gamma)(y) \sum_{l \geq 2} \frac{1}{(l-1) !} A_{\substack{\delta_{c-} \\
\gamma_{1}+\cdots+\gamma_{l}=\gamma \\
\mu_{\delta_{c}}\left(\gamma_{1}\right)=\mu\left(\gamma_{2}\right)=\cdots=\mu\left(\gamma_{l}\right)}}\left(\gamma_{1}\right) \\
& \quad \times \prod_{i=2}^{l}(-1)^{e_{i}-r(g-1)}\left[e_{i}-r_{i}(g-1)\right]_{y} H\left(\gamma_{i}\right)(y)
\end{aligned}
$$

where the sum on the right-hand side of (1.3) is finite. Moreover $\left[\delta_{c-}, \delta_{c}\right) \cap$ $\Delta\left(r_{1}, e_{1}\right)=\emptyset,\left(\delta_{c}, \delta_{c+}\right] \cap \Delta\left(r_{1}, e_{1}\right)=\emptyset$ for all $\gamma_{1}=\left(r_{1}, e_{1}\right)$ on the right-hand side of (1.3).

(ii) Let $\delta_{-}<0, \delta_{+}>0$ be noncritical stability parameters so that $\left[\delta_{-}, 0\right) \cap \Delta(r, e)=\emptyset, \quad\left(0, \delta_{+}\right] \cap \Delta(r, e)=\emptyset . \quad$ The following wallcrossing 
formula holds for $\delta_{ \pm}$sufficiently close to 0 :

$$
\begin{aligned}
& A_{\delta_{+}}(\gamma)(y)-A_{\delta_{-}}(\gamma)(y) \\
& =\sum_{l \geq 1} \frac{1}{l !} \sum_{\substack{\gamma_{1}+\cdots+\gamma_{l}=\gamma \\
\mu\left(\gamma_{i}\right)=\mu(\gamma),}} \prod_{\substack{1 \leq i \leq l \\
i=1}}^{l}(-1)^{e_{i}-r(g-1)}\left[e_{i}-r_{i}(g-1)\right]_{y} H\left(\gamma_{i}\right)(y) \\
& \quad+\sum_{l \geq 2} \frac{1}{(l-1) !} \sum_{\substack{\gamma_{1}+\cdots+\gamma_{l}=\gamma \\
\mu\left(\gamma_{i}\right)=\mu(\gamma), 1 \leq i \leq l}} A_{\delta_{-}}\left(\gamma_{1}\right)(y) \\
& \quad \times \prod_{i=2}^{l}(-1)^{e_{i}-r(g-1)}\left[e_{i}-r_{i}(g-1)\right]_{y} H\left(\gamma_{i}\right)(y)
\end{aligned}
$$

where the sum on the right-hand side of $(1.4)$ is finite. Moreover, $\left[\delta_{-}, 0\right) \cap$ $\Delta\left(r_{1}, e_{1}\right)=\emptyset,\left(0, \delta_{+}\right] \cap \Delta\left(r_{1}, e_{1}\right)=\emptyset$ for all $\gamma_{1}=\left(r_{1}, e_{1}\right)$ in the second line of the right-hand side of Equation (1.4).

Moreover $A_{\delta}(r, e) \in \mathbb{Z}\left[y, y^{-1}\right]$ if $\delta \in \mathbb{R}$ is noncritical, and $H(r, e)(y) \in$ $\mathbb{Z}\left[y, y^{-1}\right]$ if $(r, e)$ are coprime.

As mentioned above the invariants $A_{\delta}(r, e) \in \mathbb{Z}\left[y, y^{-1}\right], H(r, e)(y)$ are conjecturally related to residual equivariant Kontsevich-Soibelman invariants $\bar{A}_{\delta}(r, e)(y) \in \mathbb{Z}\left[y, y^{-1}\right], \bar{H}(r, e)(y) \in \mathbb{Z}\left[y, y^{-1}\right]$ by a refined multicover formula. For $v=1$ invariants this formula states simply that $A_{\delta}(r, e)(y)=$ $\bar{A}_{\delta}(r, e)(y)$, while the explicit formula for $v=0$ is given below.

Conjecture 1.2. Under the same hypothesis as in Conjecture 1.1, the following relation holds for any $(r, e) \in \mathbb{Z}_{\geq 1} \times \mathbb{Z}$ :

$$
H(r, e)(y)=\sum_{\substack{k \in \mathbb{Z}, k \geq 1 \\ k|r, k| e}} \frac{1}{k[k]_{y}} \bar{H}\left(\frac{r}{k}, \frac{e}{k}\right)\left(y^{k}\right) .
$$

The refined wallcrossing formulas (1.3), (1.4) are formal quantum generalizations of the wallcrossing formulas derived in [7]. Refined, or quantum, wallcrossing formulas have been physically derived in $[6,16,17]$ using arguments analogous to [10]. In particular, a refinement of the semiprimitive wallcrossing formula of [10] has been formulated in [17]. A motivic wallcrossing formula has been also announced in [41]. By analogy with [7, Section 4; 8, Section 4], the wallcrossing formulas conjectured in (1.1) can be shown to agree with the refined semiprimitive wallcrossing formulas of $[6,17,21]$, once 
the multicover formula (1.5) is properly taken into account. In particular, the above refined multicover formula can be easily inferred from $[6$, Section 4.]. The details are similar to those in [7, Section 4; 8, Section 4], hence will be omitted.

Finally, note that a refined formula has been also derived in [15] for primitive wallcrossing using arguments analogous to [10], and shown to be in a agreement with wallcrossing formulas for Poincaré and Hodge polynomials of moduli spaces of stable sheaves on surfaces [25,62,63]. The formula derived in [15] is in fact doubly refined, the BPS states being simultaneously graded by spin and $U(1)_{R^{-}}$charge quantum numbers. This motivates the following further refinement of Conjecture 1.1, which can be physically justified using arguments analogous to $[10,15,17]$.

Let $(u, v)$ be formal variables, and $\left(u^{1 / 2}, v^{1 / 2}\right)$ be formal square roots. For any $n \in \mathbb{Z}$ set

$$
[n]_{(u, v)}=\frac{(u v)^{n / 2}-(u v)^{-n / 2}}{(u v)^{1 / 2}-(u v)^{-1 / 2}} \in \mathbb{Q}\left(u^{1 / 2}, v^{1 / 2}\right) .
$$

Conjecture 1.3. Under the same conditions as in Conjecture 1.1 there exist doubly refined equivariant residual $A D H M$ invariants $A_{\delta}(r, e)(u, v) \in$ $\mathbb{Q}\left(u^{1 / 2}, v^{1 / 2}\right)$, and doubly refined Higgs sheaf invariants $H(r, e)(u, v) \in$ $\mathbb{Q}\left(u^{1 / 2}, v^{1 / 2}\right)$ so that

(i) $A_{\delta}(r, e)(u, u)=A_{\delta}(r, e)(u), H(r, e)(u, u)=H(r, e)(u)$, $A_{\delta}(r, e)(u, v) \in \mathbb{Z}\left[u^{1 / 2}, u^{-1 / 2}, v^{1 / 2}, v^{-1 / 2}\right]$ if $\delta$ is noncritical and $H(r, e)$ $(u, v) \in \mathbb{Z}\left[u^{1 / 2}, u^{-1 / 2}, v^{1 / 2}, v^{-1 / 2}\right]$ if $(r, e)$ are coprime.

(ii) $A_{\delta}(r, e)(u, v)$ satisfy wallcrossing formulas obtained by substituting $A_{\delta}\left(\gamma_{i}\right)(u, v), H\left(\gamma_{i}\right)(u, v),\left[e_{i}-r_{i}(g-1)\right]_{(u, v)} \quad$ for $\quad A_{\delta}\left(\gamma_{i}\right)(y), H\left(\gamma_{i}\right)(y)$, $\left[e_{i}-r_{i}(g-1)\right]_{y}$ in $(1.3),(1.4)$.

(iii) There exist alternative Higgs sheaf invariants $\bar{H}(r, e)(u, v) \in$ $\mathbb{Z}\left[u^{1 / 2}, u^{-1 / 2}, v^{1 / 2}, v^{-1 / 2}\right],(r, e) \in \mathbb{Z}_{\geq 1} \times \mathbb{Z}$ so that $H(r, e)(u, v), \bar{H}(r, e)$ $(u, v)$ satisfy a multicover formula obtained by making the same substitutions in (1.5).

Note that the same notation $A_{\delta}(r, e), H(r, e) ; A_{\delta}(r, e)(y), H(r, e)(y)$; $A_{\delta}(r, e)(u, v), H(r, e)(u, v)$ is (abusively) employed for rational, respectively, refined and doubly refined invariants. By convention, the distinction will reside only in the number of arguments of these rational functions. Therefore if no arguments are present, $A_{\delta}(r, e), H(r, e)$ are rational numbers, if one argument is present they are rational functions of one variable, etc. 
Moreover, the invariants $H(r, e)(y)$ will be called refined Higgs invariants in the following. The invariants $A_{\delta_{ \pm}}(r, e)(y)$ with $\delta_{ \pm}$close to 0 as in Conjecture 1.1(ii) will be denoted by $A_{0 \pm}(r, e)(y)$. Similarly the invariants $A_{\delta}(r, e)(y)$, with $\delta>\max \Delta(r, e)$, respectively, $\delta<\min \Delta(r, e)$ will be denoted by $A_{ \pm \infty}(r, e)(y)$ and referred to as asymptotic invariants.

Furthermore, note that whenever the moduli space of $\delta$-semistable objects in $\mathcal{C}_{\mathcal{X}}$ is a smooth quasi-projective variety, the corresponding doubly refined invariant coincides with its Hodge polynomial up to multiplication by a Laurent monomial in $\left(u^{1 / 2}, v^{1 / 2}\right)$. As discussed in detail in Sections $1.3,2.2$, this is the case for Higgs sheaves on $X$ with coprime numerical invariants $(r, e) \in \mathbb{Z}_{\geq 1} \times \mathbb{Z}$. If the moduli space is not smooth, the doubly refined invariant should be regarded as a virtual Hodge polynomial obtained by specializing the motivic invariant of Kontsevich and Soibelman.

Finally note that the duality isomorphisms $(2.3),(2.5)$ yield relations of the form

$$
\begin{aligned}
A_{\delta}(r, e)(y) & =A_{-\delta}(r,-e+2 r(g-1))(y), \\
H(r, e)(y) & =H(r,-e+2 r(g-1))(y)
\end{aligned}
$$

for all $(r, e) \in \mathbb{Z}_{\geq 1} \times \mathbb{Z}$. Moreover, the isomorphisms (2.6) imply that

$$
H(r, e)(y)=H(r, e+r)(y)
$$

for any $(r, e) \in \mathbb{Z}_{\geq 1} \times \mathbb{Z}$. Therefore for fixed $r$ there are only $r$ a priori distinct invariants $H(r, e)(y)$. Obviously entirely analogous formulas hold for the doubly refined invariants $A_{\delta}(r, e)(u, v), H(r, e)(u, v)$.

\subsection{Recursion formula for refined Higgs invariants}

For the purpose of the present paper, the main application of Conjectures $1.1,1.3$ is a recursion formula for the invariants $H(r, e)(y), H(r, e)(u, v)$ which determines inductively all invariants $H(r, e)(y), H(r, e)(u, v),(r, e) \in$ $\mathbb{Z}_{\geq 1} \times \mathbb{Z}$ in terms of the asymptotic invariants $A_{+\infty}(r, e)(y), A_{+\infty}(r, e)(u, v)$.

In the following $X$ is assumed to be a smooth projective curve of genus $g \geq 2$ and $p=\operatorname{deg}\left(M_{1}\right) \geq 0$. For any $\gamma=(r, e)$, let $\widetilde{\gamma}=(r,-e+2 r(g-1))$, $\widetilde{e}=-e+2 r(g-1)$. For a stability parameter $\delta$ let $\mu_{\delta}(\gamma)=(e+\delta) / r, \mu(\gamma)=$ $e / r$. Given $\gamma=\mathbb{Z} \times \mathbb{Z}$, the notation $\gamma=(r(\gamma), e(\gamma))$ will also be used on occasion.

The recursion formula will be written in detail only for the refined invariants $H(r, e)(y)$ since the analogous formula for the doubly refined invariants $H(r, e)(u, v)$ follows by obvious substitutions, as explained in Conjecture 1.3. 
Let $\gamma=(r, e) \in \mathbb{Z}_{\geq 1} \times \mathbb{Z}$ be an arbitrary numerical type. Then the following wallcrossing formula holds:

$$
\begin{aligned}
& (-1)^{e-r(g-1)}[e-r(g-1)]_{y} H(\gamma)(y)=A_{+\infty}(\gamma)(y)-A_{+\infty}(\widetilde{\gamma})(y) \\
& +\sum_{l \geq 2} \frac{(-1)^{l-1}}{(l-1) !} \sum_{\substack{\gamma_{1}, \ldots, \gamma_{l} \in \mathbb{Z}_{\geq 1} \times \mathbb{Z} \\
\gamma_{1}+\cdots,+\gamma_{l}=\gamma \\
\mu(\gamma)<\mu\left(\gamma_{i}\right), 2 \leq i \leq l}} A_{+\infty}\left(\gamma_{1}\right)(y) \prod_{i=2}^{l}(-1)^{e_{i}-r_{i}(g-1)} \\
& \times\left[e_{i}-r_{i}(g-1)\right]_{y} H\left(\gamma_{i}\right)(y) \\
& -\sum_{l \geq 2} \frac{(-1)^{l-1}}{(l-1) !} \sum_{\substack{\gamma_{1}, \ldots, \gamma_{l} \in \mathbb{Z}_{\geq 1} \times \mathbb{Z} \\
\gamma_{1}+\cdots+\gamma^{\prime}=\hat{\gamma} \\
\mu(\widetilde{\gamma}) \leq \mu\left(\gamma_{i}\right), 2 \leq i \leq l}} A_{+\infty}\left(\gamma_{1}\right)(y) \prod_{i=2}^{l}(-1)^{e_{i}-r_{i}(g-1)} \\
& \times\left[e_{i}-r_{i}(g-1)\right]_{y} H\left(\gamma_{i}\right)(y) \\
& -\sum_{l \geq 2} \frac{1}{l !} \sum_{\substack{\gamma_{1}, \ldots, \gamma_{l} \in \mathbb{Z}_{\geq 1} \times \mathbb{Z} \\
\gamma_{1}+\cdots+\gamma_{l}=\gamma \\
\mu(\gamma)=\mu\left(\gamma_{i}\right), 1 \leq i \leq l}} \prod_{i=1}^{l}(-1)^{e_{i}-r_{i}(g-1)}\left[e_{i}-r_{i}(g-1)\right]_{y} H\left(\gamma_{i}\right)(y)
\end{aligned}
$$

where the sum on the right-hand side of Equation (1.8) contains only finitely many nontrivial terms. The derivation of the recursion formula (1.8) from the wallcrossing formulas (1.3), (1.4) is presented in Section 2.4.

Remark 1.1. (i) Note that only invariants $H\left(r_{i}, e_{i}\right)(y)$ with $r_{i}<r$ enter the sum on right-hand side of (1.8). Therefore this relation completely determines all invariants $H(r, e),(r, e) \in \mathbb{Z}_{\geq 1} \times \mathbb{Z}$ if all invariants $A_{+\infty}(r, e)(y)$ are known. A conjectural formula for the asymptotic refined ADHM invariants $A_{+\infty}(r, e)(y)$ will be derived in the next section using string duality.

(ii) Given relations (1.6), (1.7), Equations (1.8) are in fact an overdetermined set of recursion relations for refined Higgs invariants. If Conjecture 1.1 holds, all these equations are compatible, and one can choose the most economical one for concrete computations. In fact, one can obtain a simpler relation by taking $e>2 r(g-1)-c(r)$ in (1.8). This results in $A_{+\infty}(\widetilde{\gamma})=0$ and the second line on the right-hand side is zero as well. However, the simpler relation obtained this way is not necessarily the most efficient as far as computer time is concerned. Concrete examples and computations will be presented in Section 4. 


\subsection{Higgs invariants and cohomology of moduli spaces of Hitchin pairs}

The goal of this subsection is to formulate one more conjecture relating refined Higgs invariants to the cohomology of moduli spaces of stable Hitchin pairs on $X$, for coprime numerical invariants $(r, e) \in \mathbb{Z} \geq \times \mathbb{Z}$. In the following it is still assumed that the genus of $X$ is $g \geq 2$, and $p=\operatorname{deg}\left(M_{1}\right) \geq 0$. Moreover, $M_{1} \simeq \mathcal{O}_{X}$ if $p=0$.

First recall that a Hitchin pair on $X$ with coefficient line bundle $L$ is a coherent sheaf $E$ equipped with a morphism $\Phi: E \rightarrow E \otimes_{X} L$. The moduli theory of such objects has been extensively and intensively studied in the mathematics literature $[5,29,54,56-58]$. In particular, as recalled in Section 2.2, there is a natural stability condition which yields an algebraic moduli stack $\mathfrak{H}(X, L, r, e)$ of finite type. Moreover, suppose $\operatorname{deg}(L) \geq$ $2 g-2$ and $L \simeq K_{X}$ if $\operatorname{deg}(L)=2 g-2$. There also exists a coarse moduli scheme $H^{\mathrm{s}}(X, L, r, e)$ parameterizing isomorphism classes of stable objects. If $(r, e) \in \mathbb{Z}_{\geq 1} \times \mathbb{Z}$ are coprime, any semistable Hitchin pair is stable, and $H^{\mathrm{s}}(X, L, r, e)$ will be denoted by $H(X, L, r, e)$.

The connection between Higgs sheaves and Hitchin pairs is based on the observation that there is a natural forgetful morphism of moduli stacks

$$
\mathfrak{H i g g} \mathfrak{g}\left(X, M_{1}, M_{2}, r, e\right) \rightarrow \mathfrak{H}\left(X, M_{2}^{-1}, r, e\right)
$$

which simply forgets $\Phi_{1}: E \otimes_{X} M_{1} \rightarrow M_{1}$. Moreover, under the current assumptions, this morphism is compatible with stability for $(r, e)$ coprime, and has a very simple structure as explained in Section 2.2. This leads to the conjecture formulated below.

First note that for $(r, e) \in \mathbb{Z}_{\geq 1} \times \mathbb{Z}$ coprime, the degree of the Poincaré polynomial $P_{y}(H(X, L, r, e))$ of the smooth moduli space $H(X, L, r, e)$ is an even integer $2 m(r, e), m(r, e) \in \mathbb{Z}_{\geq 0}$. Under the same conditions, let $H_{(u, v)}(H(X, L, r, e))$ denote the Hodge polynomial of $H(X, L, r, e)$ (see [27, Section 2]; [28, Section 2.1] for definition and properties.)

Conjecture 1.4. Under the above assumptions, let $L \simeq M_{2}^{-1}$. Then

$$
\begin{aligned}
H(r, e)(y) & =(-1)^{e-r(g-1-p)} y^{-n(r, e)} P_{(-y)}(H(X, L, r, e)) \\
H(r, e)(u, v) & =(-1)^{e-r(g-1-p)}(u v)^{-n(r, e) / 2} H_{(-u,-v)}(H(X, L, r, e))
\end{aligned}
$$

where

$$
n(r, e)=r^{2}(g-1)+r(r-1) p+m(r, e) .
$$


Remark 1.2. (i) The recursion relation (1.8) and conjecture 1.4 determine all Hodge polynomials $H_{(u, v)}(H(X, L, r, e))$ with $(r, e) \in \mathbb{Z}_{\geq 1} \times \mathbb{Z}$ coprime if the asymptotic refined ADHM invariants are known for all $(r, e) \in \mathbb{Z}_{\geq 1} \times \mathbb{Z}$. Conjectural formulas for these asymptotic invariants are presented in the next subsection.

(ii) Note that the recursion formula (1.8) determines in fact all invariants $H(r, e)(y), H(r, e)(u, v)$, including non-coprime pairs. A priori, the Higgs invariants $H(r, e)(y)$ with $(r, e)$ not coprime are not related in any direct way to the cohomology of moduli spaces of semistable Hitchin pairs with the same numerical invariants. However, a conjectural relation based on the multicover formula (1.5) will be formulated in the next subsection.

\subsection{Asymptotic refined ADHM invariants}

As explained above, the invariants $H(r, e)(y), H(r, e)(u, v)$ are completely determined by the recursion relation (1.8) if all asymptotic refined ADHM invariants are known. A conjectural formula for the generating function of asymptotic refined ADHM invariants is derived from string duality in Section 3. Basically, this generating function is determined by the Nekrasov partition function [53] of a five-dimensional supersymmetric gauge theory.

As shown below, the resulting formula involves an infinite formal sum over Young tableaus $Y$. In order to fix conventions, note that a nonempty Young tableau $Y$ is identified with a partition

$$
|Y|=Y_{1}+\cdots+Y_{l(Y)}
$$

where $|Y|$ denotes the total number of boxes of $Y$ and $l(Y)$ denotes the number of rows. For any $1 \leq i \leq l(Y), Y_{i}$ denotes the length of the $i$ th row, and $Y_{1} \geq Y_{2} \geq \cdots \geq Y_{l(Y)}$. Boxes of $Y$ will be labeled by $(i, j) \in \mathbb{Z} \times \mathbb{Z}$, $1 \leq i \leq l(Y), 1 \leq j \leq Y_{i}$.

Conjecture 1.5. Let $\mathcal{X}=\left(X, M_{1}, M_{2}\right)$ be a triple as above and let $p=$ $\operatorname{deg}\left(M_{1}\right)$. Let

$$
\mathcal{Z}_{+\infty}(\mathcal{X}, r ; \lambda, y)=\sum_{e \in \mathbb{Z}} \lambda^{e} A_{+\infty}(r, e)(y)
$$

be the generating function for the rank $r \in \mathbb{Z}_{\geq 1}$ asymptotic refined ADHM invariants conjectured in (1.1). Then

$$
Z_{+\infty}(\mathcal{X}, r ; \lambda, y)=\sum_{|Y|=r} \Omega_{Y}^{(g, p)}(\lambda, y)
$$


where

$$
\begin{aligned}
\Omega_{Y}^{(g, p)}(\lambda, y)= & (-1)^{p|Y|} y^{-p \sum_{(i, j) \in Y}(i+j-2)+(g-1) \sum_{(i, j) \in Y}\left(-2 i+2 j+1-2 Y_{i}+Y_{j}^{t}\right)} \\
& \lambda^{-p \sum_{(i, j) \in Y}(-i+j)+(g-1) \sum_{(i, j) \in Y}\left(2 i+2 j-1-2 Y_{i}-Y_{j}^{t}\right)} \\
& \prod_{(i, j) \in Y} F\left(\lambda^{-i-j+Y_{i}+Y_{j}^{t}+1} y^{i-j+Y_{i}-Y_{j}^{t}}, y\right)
\end{aligned}
$$

and

$$
F(q, z)=z^{1-g} \frac{(1-q)^{2 g}}{(1-q z)\left(1-q z^{-1}\right)} .
$$

By convention $\Omega_{\emptyset}^{(p)}(\lambda, y)=1$.

The generating function of asymptotic doubly refined ADHM invariants

$$
\mathcal{Z}_{+\infty}(\mathcal{X}, r ; \lambda, u, v)=\sum_{e \in \mathbb{Z}} \lambda^{e} A_{+\infty}(r, e)(u, v)
$$

is conjecturally determined as follows.

Conjecture 1.6. Under the same conditions as in Conjecture 1.5,

$$
Z_{+\infty}(\mathcal{X}, r ; \lambda, u, v)=\sum_{|Y|=r} \Omega_{Y}^{(g, p)}(\lambda, u, v)
$$

where

$$
\begin{aligned}
\Omega_{Y}^{(g, p)} & (\lambda, u, v) \\
= & (-1)^{p|Y|}(u v)^{-p \sum_{(i, j) \in Y}(i+j-2) / 2+(g-1) \sum_{(i, j) \in Y}\left(-2 i+2 j+1-2 Y_{i}+Y_{j}^{t}\right) / 2} \\
& \lambda^{-p \sum_{(i, j) \in Y}(-i+j)+(g-1) \sum_{(i, j) \in Y}\left(2 i+2 j-1-2 Y_{i}-Y_{j}^{t}\right)} \\
& \prod_{(i, j) \in Y} G\left(\lambda^{-i-j+Y_{i}+Y_{j}^{t}+1}(u v)^{\left(i-j+Y_{i}-Y_{j}^{t}\right) / 2},(u v)^{1 / 2},\left(u v^{-1}\right)^{1 / 2}\right)
\end{aligned}
$$

and

$$
G(q, z, w)=z^{(1-g)} \frac{(1-q w)^{g}\left(1-q w^{-1}\right)^{g}}{(1-q z)\left(1-q z^{-1}\right)} .
$$

By convention $\Omega_{\emptyset}^{(p)}(\lambda, u, v)=1$. 
Remark 1.3. Note that expression (1.15) is not obtained from Equation (1.12) by a simple substitution $y=(u v)^{1 / 2}$, as follows by comparing Equations (3.14), (3.15) in Section 3. In the first equation, the equivariant gauge theory instanton sum is evaluated at $\left(\lambda^{-1} y, \lambda y, y^{-1}\right)$ while in the second it is evaluated at $\left(\lambda^{-1}(u v)^{1 / 2}, \lambda(u v)^{1 / 2}, u^{-1}\right)$. Therefore in this case, the doubly refined invariants of Hodge type $(p, q)$ do not vanish for unequal Hodge degrees, $p \neq q$. This is in contrast with the quantum prefactors in the doubly refined refined wallcrossing formulas, which are obtained from the singly refined ones by direct substitution $y=(u v)^{1 / 2}$.

Using the recursion relation (1.8) and Conjectures (1.4-1.6), one can derive explicit formulas for the Hodge polynomials of the moduli spaces $H(X, L, r, e)$ with $(r, e)$ coprime. Note in particular that formulas (1.11), (1.14) imply that all invariants $A_{+\infty}\left(\gamma_{1}\right)(y)$ on the right-hand side of Equation (1.8) are trivial if $\mu\left(\gamma_{1}\right)<-(r-1)(2 g-2+p)$. Concrete computations are presented in Section 4 for $r=1,2,3$ and various values of $g \geq 2, p \geq 0$. In all cases, the resulting formulas are in agreement with the direct localization computations of Hitchin [29], Gothen [24] as well as the Hausel-RodriguezVillegas formula $[27,28]$. A brief survey of the results in the mathematics literature on the subject is presented in Appendix A. Moreover, direct computations in all examples considered in Section 4 support the following intriguing conjecture.

Conjecture 1.7. Under the same conditions as in Conjecture 1.5, for fixed $r \geq 1$, the refined invariants $\bar{H}(r, e)(y), \bar{H}(r, e)(u, v)$ are independent of $e \in$ $\mathbb{Z}$. In particular, they take the same value for all pairs $(r, e)$, coprime or not.

In fact, since the first version of this work was posted, the recursion relation (1.8) has been beautifully solved by Mozgovoy in [45], and the solution has been proven to be in agreement with the Hausel-Rodriguez-Villegas invariants. Furthermore, Mozgovoy's solution also satisfies the multicover formula (1.5) and has the property stated in Conjecture 1.7.

\section{ADHM invariants, Hitchin pairs and wallcrossing}

\subsection{Review of ADHM sheaves}

Let $X$ be a smooth projective curve over $\mathbb{C}$ of genus $g \geq 2$. Let $M_{1}, M_{2}$ be line bundles on $X$ so that $M_{1} \otimes_{X} M_{2} \simeq K_{X}^{-1}$, and fix such an isomorphism in the following. Let $\operatorname{deg}\left(M_{1}\right)=p, \operatorname{deg}\left(M_{2}\right)=-2 g-2-p, p \in \mathbb{Z}$ and $\mathcal{X}=$ $\left(X, M_{1}, M_{2}\right)$. 
As mentioned in the introduction, the abelian category $\mathcal{C}_{\mathcal{X}}$ of ADHM sheaves defined in [12, Section 3] consists of collections $\mathcal{E}=\left(E, V, \Phi_{1}, \Phi_{2}, \phi, \psi\right)$ on $X$ where $E$ is a coherent sheaf on $X, V$ is a finite-dimensional complex vector space, and $\Phi_{i}: E \otimes_{X} M_{i} \rightarrow E, i=1,2, \phi: E \otimes_{X} M_{1} \otimes_{X} M_{2} \rightarrow V \otimes$ $\mathcal{O}_{X}, \psi: V \otimes \mathcal{O}_{X} \rightarrow E$ are morphisms of $\mathcal{O}_{X}$-modules satisfying the ADHM relation (1.1). The morphisms of $\mathcal{C}_{\mathcal{X}}$ are natural morphisms of quiver sheaves.

An object $\mathcal{E}$ of $\mathcal{C}_{\mathcal{X}}$ will be called locally free if $E$ is a coherent locally free $\mathcal{O}_{X}$-module. Given a coherent $\mathcal{O}_{X}$-module $E$ we will denote by $r(E), d(E)$, $\mu(E)$ the rank, degree, respectively, slope of $E$ if $r(E) \neq 0$. The type of an object $\mathcal{E}$ of $\mathcal{C}_{\mathcal{X}}$ is the collection $\left.(r(\mathcal{E}), d(\mathcal{E}), v(\mathcal{E}))=(r(E), d(E), \operatorname{dim}(V))\right) \in$ $\mathbb{Z}_{\geq 0} \times \mathbb{Z} \times \mathbb{Z}_{\geq 0}$. Note that the objects of $\mathcal{C}_{\mathcal{X}}$ with $v(\mathcal{E})=0$ are triples $\mathcal{E}=$ $\left(E, \Phi_{1}, \Phi_{2}\right)$ satisfying relation (1.2), and form a full abelian subcategory of $\mathcal{C}_{\mathcal{X}}$. These are known as Higgs sheaves on $X$ with coefficient bundle $M_{1} \oplus M_{2}$ (see [12, Appendix A] for a brief summary of definitions and properties.)

The dual of a locally free $\mathrm{ADHM}$ sheaf $\mathcal{E}=\left(E, V, \Phi_{1}, \Phi_{2}, \phi, \psi\right)$ is defined by

$$
\begin{aligned}
\widetilde{E} & =E^{\vee} \otimes_{X} M^{-1} \\
\widetilde{\Phi}_{i} & =\left(\Phi_{i}^{\vee} \otimes 1_{M_{i}}\right) \otimes 1_{M^{-1}}: \widetilde{E} \otimes M_{i} \rightarrow \widetilde{E} \\
\widetilde{\phi} & =\psi^{\vee} \otimes 1_{M^{-1}}: \widetilde{E} \otimes_{X} M \rightarrow V^{\vee} \otimes \mathcal{O}_{X} \\
\widetilde{\psi} & =\phi^{\vee}: V^{\vee} \otimes \mathcal{O}_{X} \rightarrow \widetilde{E}
\end{aligned}
$$

where $i=1,2$. Obviously, if $\mathcal{E}$ is of type $(r, e, v), \widetilde{\mathcal{E}}$ is of type $(r,-e+$ $2 r(g-1), v)$.

Any real parameter $\delta \in \mathbb{R}$ determines a stability condition on $\mathcal{C}_{\mathcal{X}}[12,61]$. An object $\mathcal{E}$ of $\mathcal{C}_{\mathcal{X}}$ is $\delta$-(semi)stable if any proper nontrivial subobject $0 \subset$ $\mathcal{E}^{\prime} \subset \mathcal{E}$ satisfies the inequality

$$
r(\mathcal{E})\left(d\left(\mathcal{E}^{\prime}\right)+\delta v\left(\mathcal{E}^{\prime}\right)\right)(\leq) r\left(\mathcal{E}^{\prime}\right)(d(\mathcal{E})+\delta v(\mathcal{E}))
$$

Standard arguments show that the $\delta$-stability condition satisfies the HarderNarasimhan as well as Jordan-Hölder property for any $\delta \in \mathbb{R}$. Moreover, the following properties hold for any object $\mathcal{E}=\left(E, V, \Phi_{1}, \Phi_{2} \phi, \psi\right)$ of $\mathcal{C}_{\mathcal{X}}$ with $r(\mathcal{E}) \geq 1$ and $v(\mathcal{E})=1[12$, Section 3$]$ :

(S.1) If $\mathcal{E}$ is $\delta$-semistable for some $\delta \in \mathbb{R}$, then $\mathcal{E}$ is locally free. In addition, if $\delta>0$ then $\psi$ is not identically zero; if $\delta<0, \phi$ is not identically zero.

(S.2) If $\mathcal{E}$ is $\delta$-stable for some $\delta \in \mathbb{R}$, the endomorphism ring of $\mathcal{E}$ in $\mathcal{C}_{\mathcal{X}}$ is canonically isomorphic to $\mathbb{C}$. 
$(\mathrm{S} .3) \mathcal{E}$ is $\delta$-(semi)stable if and only if the dual $\widetilde{\mathcal{E}}$ is $(-\delta)$-(semi)stable.

One also has the following boundedness results [12, Lemmas 2.6, 2.7, Corollary 2.8]:

(B.1) The set of isomorphism classes of locally free ADHM sheaves of fixed type $(r, e, 1)$ which are $\delta$-semistable for some $\delta \in \mathbb{R}$ is bounded.

(B.2) For any $r \geq 1$ there exists an integer $c(r) \in \mathbb{Z}$ so that any $\delta$-semistable ADHM sheaf of type $(r, e, 1)$ for some $\delta>0$ satisfies $e \geq c(r)$. Note that the integer $c(r)$ is not unique unless required to be optimal with this property. In fact the proof of [12, Lemma 2.6] implies that any integer

$$
c(r) \leq-2(r-1)^{2} \max \left\{\left|\operatorname{deg}\left(M_{1}\right)\right|,\left|\operatorname{deg}\left(M_{2}\right)\right|\right\}
$$

satisfies this condition.

Note that for $v=0$ objects, $\delta$-stability is independent of $\delta$ and reduces to standard slope stability for Higgs sheaves on $X$.

A straightforward corollary of the above results is the existence of an algebraic moduli stack of finite type $\mathfrak{M}_{\delta}^{\text {ss }}(\mathcal{X}, r, e)$ of $\delta$-semistable ADHM sheaves on $X$ of type $(r, e, 1)$ for any $(r, e) \in \mathbb{Z}_{\geq 1} \times \mathbb{Z}$ and any $\delta \in \mathbb{R}$. The substack $\mathfrak{M}_{\delta}^{\mathrm{s}}(\mathcal{X}, r, e)$ of $\delta$-stable objects is separated and has the structure of a $\mathbb{C}^{\times}$-gerbe over an algebraic moduli space $M_{\delta}^{\mathrm{ss}}(\mathcal{X}, r, e)$. Property $(S .3)$ also yields a canonical isomorphism

$$
\mathfrak{M}_{\delta}^{\mathrm{ss}}(\mathcal{X}, r, e) \simeq \mathfrak{M}_{\delta}^{\mathrm{ss}}(\mathcal{X}, r,-e+2 r(g-1))
$$

for any $(r, e) \in \mathbb{Z}_{\geq 1} \times \mathbb{Z}$ and any $\delta \in \mathbb{R}$.

Moreover, there is a stability chamber structure on $\mathbb{R}_{>0}$ as follows $[12$, Section 4]. For a fixed type $(r, e) \in \mathbb{Z}_{\geq 1} \times \mathbb{Z}$, three exists a finite set $\Delta(r, e) \subset$ $\mathbb{R}_{>0}$ of critical stability parameters so that

(C.1) For any $\delta \in \mathbb{R}_{>0} \backslash \Delta(r, e), \delta$-semistability is equivalent to $\delta$-stability i.e., $\mathfrak{M}_{\delta}^{\mathrm{ss}}(\mathcal{X}, r, e)=\mathfrak{M}_{\delta}^{\mathrm{s}}(\mathcal{X}, r, e)$.

(C.2) For any $\delta>\max \Delta(\mathrm{r}, \mathrm{e}) \delta$-stability is equivalent with the following asymptotic stability condition. An object $\mathcal{E}=\left(E, V, \Phi_{i}, \phi, \psi\right)$ with $v=1$ is asymptotically stable if $E$ is locally free, $\psi$ nontrivial, and there is no proper saturated subsheaf $0 \subset E^{\prime} \subset E$ preserved by $\Phi_{i}$, $i=1,2$ so that $\operatorname{Im}(\psi) \subseteq E^{\prime}$. 
Finally note that there is a torus $\mathbf{S}=\mathbb{C}^{\times}$action on the moduli stacks $\mathfrak{M}_{\delta}^{\mathrm{ss}}(\mathcal{X}, r, e)$ so that

$$
t \times\left(E, V, \Phi_{1}, \Phi_{2}, \phi, \psi\right) \rightarrow\left(E, V, t^{-1} \Phi_{1}, t \Phi_{2}, \phi, \psi\right)
$$

on closed points. According to [12, Theorem 1.5], for noncritical stability parameter $\delta \in \mathbb{R}_{>0} \backslash \Delta(r, e)$, the stack theoretic fixed locus $\mathfrak{M}_{\delta}^{\mathrm{ss}}(\mathcal{X}, r, e)^{\mathbf{S}}$ is universally closed over $\mathbb{C}$. Moreover, the algebraic moduli space $M_{\delta}^{s s}(\mathcal{X}, r, e)$ has a perfect obstruction theory. Therefore residual $\delta$-ADHM invariants $A_{\delta}(r, e) \in \mathbb{Z}$ can be defined in each chamber by equivariant virtual localization. Wallcrossing formulas for these invariants have been derived in $[7$, Theorem 1.1] using Joyce-Song theory [38].

For future reference note that there is a completely analogous torus action on the moduli stack $\mathfrak{O b}\left(\mathcal{C}_{\mathcal{X}}\right)$ of all objects of $\mathcal{C}_{\mathcal{X}}$, which is an algebraic stack of locally finite type over $\mathbb{C}$. In particular, this yields a torus action on the moduli stack $\mathfrak{H i g h s}^{\mathrm{ss}}(\mathcal{X}, r, e)$ of slope-semistable Higgs sheaves on $X$ with fixed $(r, e) \in \mathbb{Z}_{\geq 1} \times \mathbb{Z}$, which is an algebraic stack of finite type over $\mathbb{C}$. The wallcrossing formulas in [7, Theorem 1.1] are written in terms residual equivariant generalized Donaldson-Thomas invariants $H(r, e) \in \mathbb{Q}$ defined via Joyce-Song theory applied to the stacks $\mathfrak{H i g h s}^{\mathrm{ss}}(\mathcal{X}, r, e)$. For curves $X$ of genus $g \geq 1$, the invariants $H^{\mathbf{S}}(r, e)$ are trivial, hence the wallcrossing formulas state that the invariants $A_{\delta}(r, e)$ are independent of $\delta$.

In order to conclude this section, note that the $\operatorname{stacks} \mathfrak{H i g} \mathfrak{g s}^{\mathrm{ss}}(\mathcal{X}, r, e)$ have the following simple properties. By analogy with (2.3), there is a canonical torus equivariant isomorphism

$$
\mathfrak{H i g h g}^{\mathrm{ss}}(\mathcal{X}, r, e) \simeq \mathfrak{H i g h g}^{\mathrm{ss}}(\mathcal{X}, r,-e+2 r(g-1))
$$

In addition, taking tensor product by a fixed degree one line bundle on $X$ yields an equivariant isomorphism

$$
\mathfrak{H i g h s}^{\mathrm{ss}}(\mathcal{X}, r, e) \simeq \mathfrak{H i g h}^{\mathrm{ss}}(\mathcal{X}, r, e+r)
$$

for any $(r, e) \in \mathbb{Z}_{\geq 1} \times \mathbb{Z}$. Finally note that for $(r, e)$ coprime slope semistability is equivalent to slope stability, and the stack $\mathfrak{H i g h s}^{\mathrm{ss}}(\mathcal{X}, r, e)$ has a $\mathbb{C}^{\times}$-gerbe structure over a quasi-projective scheme $\operatorname{Higgs}^{\mathrm{ss}}(\mathcal{X}, r, e)$.

\subsection{Connection with Hitchin pairs}

Let $L$ be a fixed line bundle on $X$. Recall that a Hitchin pair [29,54] on $X$ with coefficient bundle $L$ is defined as a pair $(E, \Phi)$ where $E$ is a coherent 
sheaf on $X$ and $\Phi: E \rightarrow E \otimes_{X} L$ a morphism of coherent sheaves. Such a pair is called (semi)stable if any proper nontrivial subsheaf $0 \subset E^{\prime} \subset E$ so that $\Phi\left(E^{\prime}\right) \subset E^{\prime} \otimes_{X} L$ satisfies the inequality

$$
r(E) d\left(E^{\prime}\right)(\leq) r\left(E^{\prime}\right) d(E)
$$

Note that if $r(E)>0$, semistability implies that $E$ is locally free. In the following $L$ be either $K_{X}$ or a line bundle on $X$ of degree $d(L)>2 g-2$. This will be implicitly assumed in all statements below.

Well-known results in the literature $[5,29,54,56-58]$ establish the existence of an algebraic stack of finite type $\mathfrak{H}(X, L, r, e)$ of semistable Hitchin pairs of fixed type $(r(E), d(E))=(r, e) \in \mathbb{Z}_{\geq 1} \times \mathbb{Z}$. Moreover, if $(r, e)$ are coprime, this stack is a $\mathbb{C}^{\times}$-gerbe over a smooth quasi-projective variety $H(X, L, r, e)$. For $L=K_{X}, H(X, L, r, e)$ is commonly referred to as the Hitchin integrable system.

Note that there is a torus $\mathbb{C}^{\times}$action on the stack $\mathfrak{H}(X, L, r, e)$ given by $t \times(E, \Phi) \rightarrow\left(E, t^{-1} \Phi\right)$ on closed points. The stack theoretic fixed locus is universally closed. In particular, for $(r, e)$ coprime, there is an induced torus action on the moduli scheme $H(X, L, r, e)$, and the fixed locus is a smooth projective scheme over $\mathbb{C}$.

The relation between ADHM sheaves and Hitchin pairs is summarized in the following simple observations:

(AH.1) Suppose $M_{1}=\mathcal{O}_{X}, M_{2}=K_{X}^{-1}$ and let $(r, e) \in \mathbb{Z}_{\geq 1} \times \mathbb{Z}$ be coprime. Then there is an isomorphism

$$
\mathfrak{H i g g} \mathfrak{i}(\mathcal{X}, r, e) \simeq \mathbb{C} \times \mathfrak{H}\left(X, K_{X}, r, e\right)
$$

(AH.2) Suppose $M_{2}$ is a line bundle of degree $2-2 g-p$, where $p \in \mathbb{Z}_{>0}$. Then there is an isomorphism

$$
\mathfrak{H i g h \mathfrak { g }}(\mathcal{X}, r, e) \simeq \mathfrak{H}\left(X, M_{2}^{-1}, r, e\right)
$$

Both statements rely on the fact that for coprime $(r, e)$ slope semistability is equivalent to slope stability. Therefore, the endomorphism ring of any semistable object $\mathcal{E}$ is canonically isomorphic to $\mathbb{C}$.

Then note that in the first case, given any semistable object $\mathcal{E}=\left(E, \Phi_{1}\right.$, $\Phi_{2}$ ) relation (1.2) implies that $\Phi_{1}: E \rightarrow E$ is an endomorphism of $\mathcal{E}$ since it obviously commutes with itself. Therefore it must be of the form $\Phi_{1}=\lambda 1_{E}$ for some $\lambda \in \mathbb{C}$. In particular, it preserves any subsheaf $E^{\prime} \subset E$. Generalizing 
this observation to flat families it follows that there is an forgetful morphism

$$
\mathfrak{H i g g} \mathfrak{i}(\mathcal{X}, r, e) \rightarrow \mathfrak{H}\left(X, K_{X}, r, e\right)
$$

projecting $\left(E, \Phi_{1}, \Phi_{2}\right)$ to $\left(E, \Phi_{2} \otimes 1_{K_{X}}\right)$. The isomorphism (2.8) then follows easily.

In the second case, note that given a semistable Higgs sheaf $\left(E, \Phi_{1}, \Phi_{2}\right)$, of type $(r, e)$, the data

$$
\mathcal{E}^{\prime}=\left(E \otimes X M_{1}^{-1}, \Phi_{1} \otimes 1_{M_{1}^{-1}}, \Phi_{2} \otimes 1_{M_{1}^{-1}}\right)
$$

determines a semistable Higgs sheaf of type $\left(r, e-r \operatorname{deg}\left(M_{1}\right)\right)=(r, e-r p)$. Relation (1.2) implies that $\Phi_{1} \otimes 1_{M_{1}^{-1}}$ is a morphism of (semistable) Higgs sheaves. However $\mu(\mathcal{E})>\mu\left(\mathcal{E}^{\prime}\right)$ since $p>0$, therefore any such morphism must vanish. This completes the proof.

\subsection{Remarks on refined wallcrossing conjectures}

This subsection consists of several remarks on Conjectures 1.1, 1.3. It can be skipped with no loss of essential information.

(i) First note that given any two objects $\mathcal{E}_{1}, \mathcal{E}_{2}$ of $\mathcal{C}_{\mathcal{X}}$ with $v\left(\mathcal{E}_{1}\right)+v\left(\mathcal{E}_{2}\right) \leq$ 1 , it has been proven in [12, Lemma 7.4] that the expression

$$
\begin{aligned}
& \operatorname{dim} \operatorname{Ext}_{\mathcal{C}_{\mathcal{X}}}^{0}\left(\mathcal{E}_{1}, \mathcal{E}_{2}\right)-\operatorname{dim} \operatorname{Ext}_{\mathcal{C}_{\mathcal{X}}}^{1}\left(\mathcal{E}_{1}, \mathcal{E}_{2}\right)-\operatorname{dim} \operatorname{Ext}_{\mathcal{C}_{\mathcal{X}}}^{0}\left(\mathcal{E}_{2}, \mathcal{E}_{1}\right) \\
& \quad+\operatorname{dim}_{\operatorname{Ext}_{\mathcal{C}_{\mathcal{X}}}}^{1}\left(\mathcal{E}_{2}, \mathcal{E}_{1}\right)
\end{aligned}
$$

depends only on the numerical types of the two objects. Moreover, if $\mathcal{E}_{1}, \mathcal{E}_{2}$ determine closed points in the stack theoretic fixed locus $\mathfrak{O} \mathfrak{b}\left(\mathcal{C}_{\mathcal{X}}\right)^{\mathbf{S}}$, there is an induced torus action on all the extension groups in (2.10) and the same statement holds for the alternating sum of dimensions of fixed, respectively, moving parts. This technical condition makes both Joyce-Song and Kontsevich-Soibelman theories applicable to non-Calabi-Yau categories, which is the present case.

(iii) As pointed out in [16], the quantum Donaldson-Thomas invariants of Kontsevich and Soibelman can be naturally identified with the refined topological string invariants constructed in [33] via the refined topological vertex formalism. The asymptotic invariants $A_{ \pm \infty}(r, e)(y)$ are refinements of the integral invariants $A_{ \pm \infty}(r, e)$, which are in turn identical to local stable pair invariants according to [13]. Therefore it entirely natural to expect these invariants to be determined by the refined BPS counting invariants of a local 
curve. The later can be inferred from the Nekrasov partition function of a five dimensional gauge theory as explained in Section 3.

(v) Finally note that assuming an equivariant localization result for refined invariants one can conjecture more refined wallcrossing formulas for the residual contributions of individual components of the fixed loci. This follows from the stack function relations derived in [7, Section 3].

\subsection{Derivation of recursion formula}

The purpose of this section is to prove the recursion relation (1.8), given the wallcrossing formulas (1.3), (1.4). The proof is analogous to the proof of [7, Lemma 3.8]. The main steps will be outlined below for completeness.

According to property (B.2) in Section 2.1 for any fixed $r \geq 1$ there exists an integer $c(r) \in \mathbb{Z}$ so that all invariants $A_{\delta}(r, e)(y)$, for any $\delta>0$, are identically zero if $e<c(r)$. Moreover, this integer is not unique unless required to be optimal with this property; any integer $c(r) \leq-(r-1)^{2}(2 g-$ $2+p)$ satisfies this condition. In the following set

$$
c(r)=-r(r-1)(2 g-2+p) \quad c\left(r^{\prime}\right)=-r^{\prime}(r-1)(2 g-2+p)
$$

for any $r \in \mathbb{Z}_{\geq q 1}, 1 \leq r^{\prime} \leq r$. This is not an optimal choice, but it will facilitate the derivation of formula (1.8), as shown below.

Next note that the wallcrossing formula (1.3) is equivalent to (2.12)

$$
\begin{aligned}
A_{\delta_{c}-}(\gamma)(y)-A_{\delta_{c}+}(\gamma)(y)= & \sum_{l \geq 2} \frac{(-1)^{l-1}}{(l-1) !} \sum_{\substack{\gamma_{1}+\cdots+\gamma_{l}=\gamma \\
\mu_{\delta_{c}}\left(\gamma_{1}\right)=\mu\left(\gamma_{2}\right)=\cdots=\mu\left(\gamma_{l}\right)}} A_{\delta_{c+}}\left(\gamma_{1}\right) \\
& \times \prod_{i=2}^{l}(-1)^{e_{i}-r(g-1)}\left[e_{i}-r_{i}(g-1)\right]_{y} H\left(\gamma_{i}\right) \cdot(y)
\end{aligned}
$$

For any $n \in \mathbb{Z}_{\geq 1}$ and any collection of $n$ positive integers $\left(l_{1}, \ldots, l_{n}\right) \in \mathbb{Z}_{\geq 1}^{n}$, define

$$
\begin{aligned}
\mathrm{S}_{0,+\infty}^{\left(l_{1}, \ldots, l_{n}\right)}(\gamma)=\{ & \left(\gamma_{1}, \eta_{1,1}, \ldots, \eta_{1, l_{1}}, \ldots, \eta_{n, 1}, \ldots, \eta_{n, l_{n}}\right) \\
& \in s\left(\mathbb{Z}_{\geq 1} \times \mathbb{Z}\right)^{\times\left(l_{1}+\ldots+l_{n}+1\right)} \mid \gamma_{1}+\sum_{i=1}^{n} \sum_{j=1}^{l_{i}} \eta_{i, j}=\gamma
\end{aligned}
$$




$$
\begin{aligned}
\mu_{0}(r) & \leq \mu(\gamma)<\mu\left(\eta_{1,1}\right)=\cdots=\mu\left(\eta_{1, l_{1}}\right)<\mu\left(\eta_{2,1}\right)=\cdots=\mu\left(\eta_{2, l_{2}}\right) \\
& \left.<\cdots<\mu\left(\eta_{n, 1}\right)=\cdots=\mu\left(\eta_{n, l_{n}}\right), \mu_{0}(r) \leq \mu\left(\gamma_{1}\right)\right\}
\end{aligned}
$$

where $\mu_{0}(r)=c(r) / r$. Then it straightforward to check that the union

$$
\bigcup_{n \geq 1} \bigcup_{l_{1}, \ldots, l_{n} \geq 1} \mathrm{~S}_{0,+\infty}^{\left(l_{1}, \ldots, l_{n}\right)}(\gamma)
$$

is a finite set.

Let $\left(\gamma_{1}, \eta_{1,1}, \ldots, \eta_{1, l_{1}}, \ldots, \eta_{n, 1}, \ldots, \eta_{n, l_{n}}\right) \in \mathrm{S}_{0,+\infty}^{\left(l_{1}, \ldots, l_{n}\right)}(\gamma)$ be an arbitrary element, for some $n \geq 1$ and $l_{1}, \ldots, l_{n} \geq 1$. Let $\mu_{i}, 1 \leq i \leq n$ denote the common value of the slopes $\mu\left(\eta_{i, j}\right), 1 \leq j \leq l_{i}$. If $n \geq 2$, let also

$$
\gamma_{n-i+2}=\gamma_{1}+\eta_{i, 1}+\cdots+\eta_{n, l_{n}}
$$

for $2 \leq i \leq n$. Define the stability parameters $\delta_{i}, 1 \leq i \leq n$ by

$$
\begin{aligned}
& \mu_{\delta_{1}}\left(\gamma_{1}\right)=\mu_{n} \\
& \mu_{\delta_{i}}\left(\gamma_{i}\right)=\mu_{n+1-i}, \quad 2 \leq i \leq n \quad(\text { if } n \geq 2) .
\end{aligned}
$$

By construction, $\delta_{i}$ is a critical stability parameter of type $\gamma_{i}$ for all $1 \leq i \leq n$. Given the slope inequalities in (2.13), it is straightforward to check that

$$
0<\delta_{n}<\delta_{n-1}<\cdots<\delta_{1}
$$

Moreover, $\mu\left(\gamma_{i}\right) \geq \mu_{0}(r)$ for all $1 \leq i \leq n$ since the integers $c\left(r^{\prime}\right), 1 \leq r^{\prime} \leq r$ defined in (2.11) satisfiy

$$
\frac{c\left(r^{\prime}\right)}{r^{\prime}}=-(r-1)(2 g-2+p)=\mu_{0}(r) .
$$

Next note that the set $\Delta_{\gamma}$ of all stability parameters constructed this way, for all $n \geq 1$ and any possible values of $l_{1}, \ldots, l_{n}$ is finite, since the set (2.14) is finite. Therefore one can choose stability parameters $0<\delta_{0+}<$ $\min \Delta_{\gamma}, \delta_{+\infty}>\max \Delta_{\gamma}$. By construction $\Delta_{\gamma}$ contains all possible decreasing finite sequences of stability parameters of the form (2.16) with the property that there exists

$$
\left(\gamma_{1}, \eta_{1,1}, \ldots, \eta_{1, l_{1}}, \ldots, \eta_{n, 1}, \ldots, \eta_{n, l_{n}}\right) \in\left(\mathbb{Z}_{\geq} \times \mathbb{Z}\right)^{\times\left(l_{1}+\cdots+l_{n}+1\right)}
$$

for some $l_{1}, \ldots, l_{n} \geq 1$ so that 
(a) $\gamma_{1}+\eta_{1,1}+\cdots+\eta_{n, l_{n}}=\gamma$

(b) Conditions (2.15) hold.

In conclusion, successive applications of the wallcrossing formula (2.12) yield (2.18)

$$
\begin{aligned}
& A_{0+}(\gamma)-A_{+\infty}(\gamma) \\
& =\sum_{n=1}^{\infty} \sum_{l_{1}, \ldots, l_{n} \geq 1} \prod_{i=1}^{n} \frac{(-1)^{l_{i}}}{l_{i} !} \sum_{\substack{\mu_{1}+\eta_{1,1}+\cdots+\eta_{1, l_{1}}+\cdots+\eta_{n, 1}+\cdots+\eta_{n, l_{n}}=\gamma, \mu_{0}(r) \leq \mu(\gamma)<\mu\left(\eta_{1,1}\right)=\cdots \mu\left(\eta_{1, l_{1}}\right)<\cdots<\mu\left(\eta_{n, 1}\right)=\cdots=\mu\left(\eta_{n, l_{n}}\right) \\
\mu_{0}(r) \leq \mu\left(\gamma_{1}\right)}} \\
& A_{+\infty}\left(\gamma_{1}\right)(y) \prod_{i=1}^{n} \prod_{j=1}^{l_{i}}(-1)^{e_{i, j}-r_{i, j}(g-1)}\left[e_{i, j}-r_{i, j}(g-1)\right]_{y} H\left(\eta_{i, j}\right)(y)
\end{aligned}
$$

where $\gamma=\left(r_{1}, e_{1}\right) \in \mathbb{Z}_{\geq 1} \times \mathbb{Z}, \eta_{i, j}=\left(e_{i, j}, r_{i, j}\right) \in \mathbb{Z}_{\geq 1} \times \mathbb{Z}, 1 \leq i \leq n, 1 \leq j \leq$ $l_{i}$. T Moreover, the sum on the right-hand side of Equation (2.18) is finite for any fixed $\gamma=(r, e)$.

Then in Equation (1.4) $A_{\delta_{-}}(\gamma)=A_{\delta_{+}}(\widetilde{\gamma})$ and

$$
\begin{aligned}
\sum_{l \geq 2} & \frac{1}{(l-1) !} \sum_{\substack{\gamma_{1}+\cdots+\gamma_{l}=\gamma \\
\mu\left(\gamma_{i}\right)=\mu(\gamma), 1 \leq i \leq l}} A_{\delta_{-}}\left(\gamma_{1}\right)(y) \prod_{i=2}^{l-1} \mathrm{e}^{e_{i}-r_{i}(g-1)}\left[e_{i}-r_{i}(g-1)\right]_{y} H\left(\gamma_{i}\right)(y) \\
= & \sum_{l \geq 2} \frac{1}{(l-1) !} \sum_{\substack{\gamma_{1}+\cdots+\gamma_{l}=\gamma \\
\mu\left(\gamma_{i}\right)=\mu(\gamma), 1 \leq i \leq l}} A_{\delta_{+}}\left(\widetilde{\gamma}_{1}\right)(y) \prod_{i=2}^{l-1} \mathrm{e}^{e_{i}-r_{i}(g-1)}\left[e_{i}-r_{i}(g-1)\right]_{y} \\
= & \sum_{l \geq 2} \frac{(-1)^{l-1}}{(l-1) !} \sum_{\substack{\mu\left(\gamma_{i}\right)=\mu(\widetilde{\gamma}), 1 \leq i \leq l \\
\gamma_{1}+\cdots+\gamma_{l}=\widetilde{\gamma}}} A_{\delta_{+}}\left(\gamma_{1}\right)(y) \prod_{i=2}^{l-1} \mathrm{e}^{e_{i}-r_{i}(g-1)} \\
& \times\left[e_{i}-r_{i}(g-1)\right]_{y} H\left(\gamma_{i}\right)(y)
\end{aligned}
$$

by a redefinition of variables. Substituting (2.18) and (2.19) in Equation (1.4), Equation (1.8) follows by simple combinatorics.

\section{Asymptotic refined ADHM invariants from gauge theory}

The main goal of this section is to present a string theoretic derivation of Conjecture 1.5. Readers who are not interested in this derivation are encouraged to skip this section. 
Conjecture 1.5 will be shown to follow from type IIA/M-theory duality using arguments analogous to [19,20,30-33,40,43,44,53]. Summarizing these results, the topological string amplitudes of certain toric Calabi-Yau threefolds (as well as some nontoric configurations of local rational curves) were identified with the instanton partition function of five-dimensional gauge theory compactified on a circle of finite radius. The later has been identified in [53] with the generating function for the equivariant Hirzebruch genus of the moduli space of torsion free framed sheaves on the projective plane. A mathematical exposition can be found for example in $[51,52]$. The relation between topological strings and five-dimensional gauge theory has been subsequently refined in [33]. Moreover, the refined topological string partition function constructed in [33] has been conjecturally identified in [16] with the generation function of refined Donaldson-Thomas invariants. The present problem requires a version of this identification for higher genus local curves.

\subsection{Geometric engineering via local ruled surfaces}

Working under the same assumptions as in Section 2.2, $M_{1}, M_{2}$ are line bundles on the curve $X$ so that $M_{1} \otimes_{X} M_{2} \simeq K_{X}^{-1}, p=d\left(M_{1}\right) \geq 0$ and $M_{1} \simeq \mathcal{O}_{X}$ if $p=0$. Let $Y$ be the total space of the rank two vector bundle $M_{1}^{-1} \oplus M_{2}^{-1}$ on $X$, which is a noncompact Calabi-Yau three-fold under the current assumptions. There is a torus action $\mathbf{S} \times Y \rightarrow Y$ scaling $M_{1}^{-1}$, $M_{2}^{-1}$ with characters $t, t^{-1}$, so that $Y$ is equivariantly $\mathrm{K}$-trivial. In principle, the relevant five-dimensional gauge theory should be constructed by geometric engineering, that is identifying the low-energy effective action of an M-theory supersymmetric background defined by $S^{1} \times Y$. This direct approach is somewhat problematic in the present case. A much clearer picture emerges considering a different local Calabi-Yau threefold constructed as follows.

Let $S$ be the Total space of the projective bundle $\mathbb{P}\left(\mathcal{O}_{X} \oplus M_{1}\right)$. $S$ is a smooth geometrically ruled surface over $X$ and it has two canonical sections $X_{1}, X_{2}$ with normal bundles

$$
N_{X_{1} / S} \simeq M_{1}^{-1}, \quad N_{X_{2} / S} \simeq M_{1}
$$

respectively. Note that the cone of effective curve classes on $S$ is generated by the section class $\left[X_{2}\right]$ and the fiber class. 
Let $Z$ be the Total space of the canonical bundle $K_{S}$, which is again a noncompact Calabi-Yau three-fold. The normal bundle to $X_{1}$ in $Z$ is

$$
N_{X_{1} / Z} \simeq M_{1}^{-1} \oplus K_{X} \otimes_{X} M_{1} \simeq M_{1}^{-1} \otimes M_{2}^{-1}
$$

therefore the total space of $N_{X_{1} / Z}$ is isomorphic to $Y$. Moreover, there is a torus action $\mathbf{S} \times Z \rightarrow Z$ so that $Z$ is equivariantly Calabi-Yau and the induced torus action on $N_{X_{1} / Z}$ is compatible with the torus action on $Y$.

Now the main observation is that the local three-fold $Z$ engineers a supersymmetric five-dimensional $S U(2)$ gauge theory with $g$ adjoint hypermultiplets on $\mathbb{C}^{2} \times S^{1}$, where $g$ is the genus of $X$ [39]. The integer $p=\operatorname{deg}\left(M_{1}\right)$ corresponds to the level of the five-dimensional Chern-Simons term [59]. Therefore by analogy with [19,20,30-33,40,43,44,53], the refined topological string partition function of $Z$ should be related with the equivariant instanton partition function $\mathcal{Z}_{\text {inst }}^{(p)}\left(Q, \epsilon_{1}, \epsilon_{2}, a_{1}, a_{2}, y\right)$, which has been constructed in [53]. As explained in detail in the next subsection, $\mathcal{Z}_{\text {inst }}^{(p)}\left(Q, \epsilon_{1}, \epsilon_{2}, a_{1}, a_{2}, y\right)$ is the generating function for the $\chi_{y}$-genus of a certain holomorphic bundle on a partial compactification of the instanton moduli space. In particular $\epsilon_{1}, \epsilon_{2}, a_{1}, a_{2}$ are equivariant parameters for a natural torus action, $Q$ is a formal variable counting instanton charge and $y$ is another formal variable.

In order to make string duality predictions more precise, let $Q_{\mathrm{f}}, Q_{\mathrm{b}}$ be formal symbols associated to the fiber class, respectively, section class $\left[X_{1}\right]$ on $Z$. Then string duality predicts that there is a factorization

$$
\mathcal{Z}_{\mathrm{ref}}\left(Z ; Q_{\mathrm{f}}, Q_{\mathrm{b}}, q, y\right)=\mathcal{Z}_{\text {ref }}^{\text {pert }}\left(Z ; Q_{\mathrm{f}}, q, y\right) \mathcal{Z}_{\text {ref }}^{\text {nonpert }}\left(Z ; Q_{\mathrm{f}}, Q_{\mathrm{b}}, q, y\right)
$$

into a perturbative, respectively, nonperturbative parts. Moreover, and there is an identification

$$
\mathcal{Z}_{\text {ref }}^{\text {nonpert }}\left(Z ; Q_{\mathrm{f}}, Q_{\mathrm{b}}, q, y\right)=\mathcal{Z}_{\text {inst }}^{(p)}\left(Q, \epsilon_{1}, \epsilon_{2}, a_{1}, a_{2}, y\right)
$$

subject to certain duality relations between the formal parameters in the two partition functions.

Next note that only nonnegative powers of $Q_{\mathrm{b}}, Q_{\mathrm{f}}$ can appear in $\mathcal{Z}_{\text {ref }}(Z$; $\left.Q_{\mathrm{f}}, Q_{\mathrm{b}}, q, y\right)$ since the section class $\left[X_{1}\right]$ and the fiber class generate the Mori cone of $S$. Similarly, only nonnegative powers of $Q_{\mathrm{f}}$ can appear in $\mathcal{Z}_{\text {ref }}^{\text {pert }}\left(Z ; Q_{\mathrm{f}}, q, y\right)$, which represents the contribution of pure fiber classes to $\mathcal{Z}_{\text {ref }}\left(Z ; Q_{\mathrm{f}}, Q_{\mathrm{b}}, q, y\right)$. Therefore $\mathcal{Z}_{\text {ref }}\left(Z ; Q_{\mathrm{f}}, Q_{\mathrm{b}}, q, y\right), \mathcal{Z}_{\text {ref }}^{\text {pert }}\left(Z ; Q_{\mathrm{f}}, q, y\right)$ have well-defined specialization at $Q_{\mathrm{f}}=0$. Moreover, by construction $\left.\mathcal{Z}_{\text {ref }}^{\text {pert }}\left(Z ; Q_{\mathrm{f}}, q, y\right)\right|_{Q_{\mathrm{f}}=0}=1$. Therefore $\mathcal{Z}_{\text {ref }}^{\text {nonpert }}\left(Z ; Q_{\mathrm{f}}, Q_{\mathrm{b}}, q, y\right)$ has welldefined specialization at $Q_{\mathrm{f}}=0$ as well, which is determined by the instanton 
expansion $\mathcal{Z}_{\text {inst }}^{(p)}\left(Q, \epsilon_{1}, \epsilon_{2}, a_{1}, a_{2}, y\right)$. The refined theory of the local three-fold $Y$ is then determined by identifying the contributions of curves supported on the section $X_{1}$ to $\left.\mathcal{Z}_{\text {ref }}^{\text {nonpert }}\left(Z ; Q_{\mathrm{f}}, Q_{\mathrm{b}}, q, y\right)\right|_{Q_{\mathrm{f}}=0}$. Computations will be carried out in detail in the next subsections, resulting in explicit formulas for the instanton partition function and duality relations among formal variables.

\subsection{Hirzebruch genus}

Let $M(r, k)$ denote the moduli space of rank $r$ framed torsion-free sheaves $(F, f)$ on $\mathbb{P}^{2}$ with second Chern class $k \in \mathbb{Z}_{\geq 0}$. The framing data is an isomorphism

$$
f:\left.F\right|_{\mathbb{P}_{\infty}^{1}} \rightarrow \mathcal{O}_{\mathbb{P}_{\infty}^{1}}^{\oplus r}
$$

$M(r, k)$ is a smooth quasi-projective fine moduli space i.e., there is an universal framed sheaf $(\mathrm{F}, \mathrm{f})$ on $M(r, k) \times \mathbb{P}^{2}$. Let $\mathrm{V}=R^{1} p_{1 *} \mathrm{~F} \otimes p_{2}^{*} \mathcal{O}_{\mathbb{P}^{2}}(-1)$ where $p_{1}, p_{2}: M(r, k) \times \mathbb{P}^{2} \rightarrow M(r, k), \mathbb{P}^{2}$ denote the canonical projections. It follows from [50] that $\mathrm{V}$ is a locally free sheaf of rank $k$ on $M(r, k)$.

There is a torus $\mathbf{T}=\mathbb{C}^{\times} \times \mathbb{C}^{\times} \times\left(\mathbb{C}^{\times}\right)^{\times r}$ action on acting on $M(r, k)$, where the action of the first two factors is induced by the canonical action on $\mathbb{C}^{\times} \times \mathbb{C}^{\times}$on $\mathbb{P}^{2}$, and the last $r$ factors act linearly on the framing. According to [51] the fixed points of the $\mathbf{T}$-action on $M(r, k)$ are isolated and classified by collections of Young diagrams $\underline{Y}=\left(Y_{1}, \ldots, Y_{r}\right)$ so that the total number of boxes in all diagrams is $|\underline{Y}|=\left|Y_{1}\right|+\cdots+\left|Y_{r}\right|=k$. Let $\mathcal{Y}_{r, k}$ denote the set of all such $r$-uples of Young diagrams. Note also that both the holomorphic cotangent bundle $T_{M(r, k)}^{\vee}$ and the bundle $\mathrm{V}$ constructed in the previous paragraph carry canonical equivariant structures.

The K-theoretic instanton partition function of an $S U(2)$ theory with $g$ adjoint hypermultiplets and a level $p$ Chern-Simons term is given by the equivariant residual Hirzebruch genus of the holomorphic T-equivariant bundle

$$
\left(T_{M(2, k)}^{\vee}\right)^{\oplus g} \otimes(\operatorname{det} \mathrm{V})^{-p}
$$

This is defined by equivariant localization as follows [44,52]. Let $\left(\epsilon_{1}, \epsilon_{2}, a_{1}, a_{2}\right)$ be equivariant parameters associated to the torus $\mathbf{T}$. Then the localization formula yields $[44,52]$

$$
\mathcal{Z}_{\text {inst }}^{(g, p)}\left(Q, \epsilon_{1}, \epsilon_{2}, a_{1}, a_{2}, y\right)=\sum_{k=0}^{\infty} Q^{k} \mathcal{Z}_{k}^{(g, p)}\left(\epsilon_{1}, \epsilon_{2}, a_{1}, a_{2} ; y\right)
$$


where $\mathcal{Z}_{0}^{(g, p)}\left(\epsilon_{1}, \epsilon_{2}, a_{1}, a_{2} ; y\right)=1$ and

$$
\begin{aligned}
\mathcal{Z}_{k}^{(g, p)}\left(\epsilon_{1}, \epsilon_{2}, a_{1}, a_{2} ; y\right)= & \sum_{\underline{Y} \in \mathcal{Y}_{2, k}} \prod_{\alpha=1}^{2}\left(\mathrm{e}^{-\left|Y_{\alpha}\right| a_{\alpha}} \prod_{(i, j) \in Y_{\alpha}} \mathrm{e}^{(i-1) \epsilon_{1}+(j-1) \epsilon_{2}}\right)^{p} \\
& \prod_{\alpha, \beta=1}^{2} \prod_{(i, j) \in Y_{\alpha}} \frac{\left(1-\mathrm{ye}^{\left(Y_{\beta, j}^{\mathrm{t}}-i\right) \epsilon_{1}-\left(Y_{\alpha, i}-j+1\right) \epsilon_{2}+a_{\alpha \beta}}\right)^{g}}{\left(1-\mathrm{e}^{\left(Y_{\beta, j}^{\mathrm{t}}-i\right) \epsilon_{1}-\left(Y_{\alpha, i}-j+1\right) \epsilon_{2}+a_{\alpha \beta}}\right)} \\
& \prod_{(i, j) \in Y_{\beta}} \frac{\left(1-y \mathrm{e}^{-\left(Y_{\alpha, j}^{\mathrm{t}}-i+1\right) \epsilon_{1}+\left(Y_{\beta, i}-j\right) \epsilon_{2}+a_{\alpha \beta}}\right)^{g}}{\left(1-\mathrm{e}^{-\left(Y_{\alpha, j}^{\mathrm{t}}-i+1\right) \epsilon_{1}+\left(Y_{\beta, i}-j\right) \epsilon_{2}+a_{\alpha \beta}}\right)}
\end{aligned}
$$

where for any Young tableau $Y, Y_{i}, i \in \mathbb{Z}_{\geq 1}$ denotes the length of the $i$ th column and $Y^{t}$ denotes the transpose of $Y$. If $i$ is greater than the number of columns of $Y, Y_{i}=0$. Moreover $a_{\alpha \beta}=a_{\alpha}-a_{\beta}$ for any $\alpha, \beta=1,2$.

\subsection{Comparison with the ruled vertex}

A conjectural formula for the unrefined topological string partition function $\mathcal{Z}_{\text {top }}\left(Z ; Q_{\mathrm{f}}, Q_{\mathrm{b}}, q\right)$ of the three-fold $Z$ has been derived from large $N$ duality in [14]. The purpose of this subsection is to show that $\mathcal{Z}_{\text {top }}\left(Z ; Q_{\mathrm{f}}, Q_{\mathrm{b}}, q\right)$ has a factorization of the form (3.1) and there is an identification

$$
\mathcal{Z}_{\text {top }}^{\text {nonpert }}\left(Z ; Q_{\mathrm{f}}, Q_{\mathrm{b}}, q\right)=\mathcal{Z}_{\text {inst }}^{(g, p)}\left(Q, \epsilon_{1}, \epsilon_{2}, a_{1}, a_{2}, y\right)
$$

subject to certain duality relations between the formal parameters. This will be a confirmation of duality predictions for local ruled surfaces in the unrefined case. Moreover, it will provide a starting point for understanding this correspondence in the refined case.

By analogy with $[31,44]$, first set

$$
-\epsilon_{1}=\epsilon_{2}=\hbar, \quad y=1 \text {. }
$$

Then a straightforward computation yields

$$
\begin{aligned}
& \mathcal{Z}_{2, k}^{(g, p)}\left(-\hbar, \hbar, a_{1}, a_{2}, 1\right) \\
& \quad=\sum_{\substack{Y_{1}, Y_{2} \\
\left|Y_{1}\right|+\left|Y_{2}\right|=k}} \mathrm{e}^{-p\left(\left|Y_{1}\right| a_{1}+\left|Y_{2}\right| a_{2}\right)} \prod_{\alpha=1}^{2} \prod_{(i, j) \in Y_{\alpha}} \mathrm{e}^{p(j-i) \hbar}
\end{aligned}
$$




$$
\begin{aligned}
& \times\left(2 \sinh \frac{\hbar}{2}\left(Y_{\alpha, i}+Y_{\alpha, j}^{\mathrm{t}}-i-j+1\right)\right)^{2(g-1)} \\
& \prod_{(i, j) \in Y_{1}}\left(2 \sinh \frac{1}{2}\left(a_{1,2}+\left(Y_{2, j}^{\mathrm{t}}+Y_{1, i}-i-j+1\right) \hbar\right)\right)^{2(g-1)} \\
& \prod_{(i, j) \in Y_{2}}\left(2 \sinh \frac{1}{2}\left(a_{1,2}-\left(Y_{1, j}^{\mathrm{t}}+Y_{2, i}-i-j+1\right) \hbar\right)\right)^{2(g-1)}
\end{aligned}
$$

Using identity [44, Lemma 4.4], which was conjectured in [31] and proven in $[20]$, it follows that

$$
\begin{aligned}
\mathcal{Z}_{2, k}^{(g, p)}\left(-\hbar, \hbar, a_{1}, a_{2}, 1\right) & =\sum_{\substack{Y_{1}, Y_{2} \\
\left|Y_{1}\right|+\left|Y_{2}\right|=k}} 2^{8(g-1)\left(\left|Y_{1}\right|+\left|Y_{2}\right|\right)} \mathrm{e}^{-p\left(\left|Y_{1}\right| a_{1}+\left|Y_{2}\right| a_{2}\right)} \mathrm{e}^{p\left(\kappa\left(Y_{1}\right)+\kappa\left(Y_{2}\right)\right) \hbar / 2} \\
& \prod_{\alpha, \beta=1}^{2} \prod_{i, j=1}^{\infty}\left(\frac{\sinh \frac{1}{2}\left(a_{\alpha, \beta}+\left(Y_{\alpha, i}-Y_{\beta, j}+j-i\right) \hbar\right)}{\sinh \frac{1}{2}\left(a_{\alpha, \beta}+(j-i) \hbar\right)}\right)^{2(1-g)}
\end{aligned}
$$

where for any Young diagram $Y$

$$
\kappa(Y)=2 \sum_{(i, j) \in Y}(j-i)=|Y|+\sum_{i=1}^{l(Y)}\left(Y_{i}^{2}-2 i Y_{i}\right)
$$

$l(Y)$ being the number of rows of $Y$. Note that $\kappa(Y)=-\kappa\left(Y^{\mathrm{t}}\right)$.

The topological string partition function on $Z$ computed by the ruled vertex formalism [14] is

$$
\begin{aligned}
\mathcal{Z}_{\text {top }}\left(Z ; q, Q_{\mathrm{f}}, Q_{\mathrm{b}}\right)= & \sum_{Y_{1}, Y_{2}}\left(K_{Y_{1}, Y_{2}}\left(q, Q_{\mathrm{f}}\right)\right)^{2(1-g)} Q_{\mathrm{b}}^{\left|Y_{1}\right|+\left|Y_{2}\right|} Q_{\mathrm{f}}^{p\left|Y_{2}\right|}(-1)^{p\left(\left|Y_{1}\right|+\left|Y_{2}\right|\right)} \\
& \times q^{p\left(\kappa\left(Y_{2}\right)-\kappa\left(Y_{1}\right)\right) / 2}
\end{aligned}
$$

where

$$
K_{Y_{1}, Y_{2}}\left(q, Q_{\mathrm{f}}\right)=\sum_{Y} Q_{\mathrm{f}}^{|Y|} W_{Y_{2} Y}(q) W_{Y Y_{1}}(q)
$$

and

$$
\left.W_{R_{1}, R_{2}}(q)=s_{R_{2}}\left(q^{-i+1 / 2}\right)\right) s_{R_{1}}\left(q^{R_{2, i}-i+1 / 2}\right)
$$


for any two Young tableaus $R_{1}, R_{2}$. Here $s_{R}\left(x^{i}\right)$ denotes the Schur function associated to the Young tableau $R$.

According to $[20,31$; Theorem $7.1,44], K_{Y_{1}, Y_{2}}\left(q, Q_{\mathrm{f}}\right)=K_{Y_{2}, Y_{1}}\left(q, Q_{\mathrm{f}}\right)$ and

$$
\begin{aligned}
\frac{K_{Y_{1}, Y_{2}^{\mathrm{t}}}\left(\mathrm{e}^{-z}, \mathrm{e}^{-b}\right)}{K_{\emptyset, \emptyset}\left(\mathrm{e}^{-z}, \mathrm{e}^{-b}\right)}= & \left(2^{-4} Q_{\mathrm{f}}^{-1 / 2}\right)^{\left|Y_{1}\right|+\left|Y_{2}\right|} \prod_{\alpha, \beta=1}^{2} \prod_{i, j=1}^{\infty} \\
& \times \frac{\sinh \frac{1}{2}\left(b_{\alpha, \beta}+\left(Y_{\alpha, i}-Y_{\beta, j}+j-i\right) z\right)}{\sinh \frac{1}{2}\left(b_{\alpha, \beta}+(j-i) z\right)} .
\end{aligned}
$$

where $b_{1,2}=-b_{2,1}=b$. Therefore (3.8) is equivalent to

$$
\begin{aligned}
\mathcal{Z}_{\text {top }}\left(Z ; q, Q_{\mathrm{f}}, Q_{\mathrm{b}}\right)= & \sum_{Y_{1}, Y_{2}}\left(K_{Y_{2}, Y_{1}^{\mathrm{t}}}\left(q, Q_{\mathrm{f}}\right)\right)^{2(1-g)} Q_{\mathrm{b}}^{\left|Y_{1}\right|+\left|Y_{2}\right|} Q_{\mathrm{f}}^{p\left|Y_{2}\right|}(-1)^{p\left(\left|Y_{1}\right|+\left|Y_{2}\right|\right)} \\
& \times q^{p\left(\kappa\left(Y_{1}\right)+\kappa\left(Y_{2}\right)\right) / 2}
\end{aligned}
$$

Setting

$$
\begin{aligned}
\mathcal{Z}_{\text {top }}^{\text {pert }}\left(Z ; q, Q_{\mathrm{f}}, Q_{\mathrm{b}}\right) & =K_{\emptyset, \emptyset}\left(q, Q_{\mathrm{f}}\right)^{2(1-g)}, \\
\mathcal{Z}_{\text {top }}^{\text {nonpert }}\left(Z ; q, Q_{\mathrm{f}}, Q_{\mathrm{b}}\right) & =\frac{\mathcal{Z}_{\text {top }}\left(q, Q_{\mathrm{f}}, Q_{\mathrm{b}}\right)}{K_{\emptyset, \emptyset}\left(q, Q_{\mathrm{f}}\right)^{2(1-g)}} .
\end{aligned}
$$

Identity (3.9) yields

$$
\mathcal{Z}_{\text {top }}^{\text {nonpert }}\left(Z ; q, Q_{\mathrm{f}}, Q_{\mathrm{b}}\right)=\sum_{k=0}^{\infty} Q^{k} \mathcal{Z}_{2, k}^{(g, p)}\left(-\hbar, \hbar, a_{1}, a_{2} ; 1\right)
$$

for the following change of variables:

$$
Q_{\mathrm{f}}=\mathrm{e}^{a_{12}}, \quad q=\mathrm{e}^{\hbar}, \quad Q=Q_{\mathrm{b}} Q_{\mathrm{f}}^{g-1}, \quad \mathrm{e}^{a_{1}}=-1
$$

This is a concrete confirmation of duality predictions in the unrefined case. The refined case is the subject of the next subsection.

\subsection{Refinement}

As explained at the end of Section 3.1, string duality predicts that the nonperturbative part of the refined topological partition function of $Z$ is determined by instanton partition function $\mathcal{Z}_{\text {inst }}^{(p)}\left(Q, \epsilon_{1}, \epsilon_{2}, a_{1}, a_{2}, y\right)$ provided 
one finds the correct identification of formal parameters as in [30,33]. Although local ruled surfaces are not discussed in [30,33], a careful inspection of the cases discussed there leads to the following construction.

Recall that the contribution of a fixed point $\left(Y_{1}, Y_{2}\right) \in \mathcal{Y}_{2, k}$ for some arbitrary $k \geq 1$ to the right-hand side of the localization formula (3.4) is

$$
\begin{aligned}
& \prod_{\alpha=1}^{2}\left(\mathrm{e}^{-\left|Y_{\alpha}\right| a_{\alpha}} \prod_{(i, j) \in Y_{\alpha}} \mathrm{e}^{(i-1) \epsilon_{1}+(j-1) \epsilon_{2}}\right)^{p} \\
& \prod_{\alpha, \beta=1}^{2} \prod_{(i, j) \in Y_{\alpha}} \frac{\left(1-y \mathrm{e}^{\left.\left(Y_{\beta, j}^{\mathrm{t}}-i\right) \epsilon_{1}-\left(Y_{\alpha, i}-j+1\right) \epsilon_{2}+a_{\alpha}-a_{\beta}\right)^{g}}\right.}{\left(1-\mathrm{e}^{\left.\left(Y_{\beta, j}^{\mathrm{t}}-i\right) \epsilon_{1}-\left(Y_{\alpha, i}-j+1\right) \epsilon_{2}+a_{\alpha}-a_{\beta}\right)}\right.} \\
& \prod_{(i, j) \in Y_{\beta}} \frac{\left(1-y \mathrm{e}^{-\left(Y_{\alpha, j}^{\mathrm{t}}-i+1\right) \epsilon_{1}+\left(Y_{\beta, i}-j\right) \epsilon_{2}+a_{\alpha}-a_{\beta}}\right)^{g}}{\left(1-\mathrm{e}^{\left.-\left(Y_{\alpha, j}^{\mathrm{t}}-i+1\right) \epsilon_{1}+\left(Y_{\beta, i}-j\right) \epsilon_{2}+a_{\alpha}-a_{\beta}\right)}\right.}
\end{aligned}
$$

Let $\mathcal{Z}_{(\emptyset, Y)}^{(g, p)}\left(q_{1}, q_{2}, Q_{\mathrm{f}}, y\right)$ be the expression obtained by setting $q_{1}=\mathrm{e}^{-\epsilon_{1}}, q_{2}=$ $\mathrm{e}^{-\epsilon_{2}}$ and

$$
Q_{\mathrm{f}}=\mathrm{e}^{a_{12}}, \quad \mathrm{e}^{a_{1}}=-1
$$

in (3.13). Note that a simple power counting argument shows that the expression

$$
Q_{\mathrm{f}}^{(g-1)|Y|} \mathcal{Z}_{(Y, \emptyset)}^{(g, p)}\left(q_{1}, q_{2}, Q_{\mathrm{f}}, y\right)
$$

has well-defined specialization $\mathcal{Z}_{(Y, \emptyset)}^{(g, p)}\left(q_{1}, q_{2}, y\right)^{(0)}$ at $Q_{\mathrm{f}}=0$, for any $Y$. Then, for any $r \in \mathbb{Z}_{\geq 1}$, any Young diagram $Y$ with $|Y|=r$, and any $p \in \mathbb{Z}$ let

$$
\Omega_{Y}^{(g, p)}(\lambda, y)=y^{2|Y|} \lambda^{(g-1)|Y|} \mathcal{Z}_{(Y, \emptyset)}^{(g, p)}\left(\lambda^{-1} y, \lambda y, y^{-1}\right)^{(0)}
$$

Then string duality predicts that the generating function of asymptotic singly refined ADHM invariants is given by

$$
\mathcal{Z}_{+\infty}(\mathcal{X}, r ; \lambda, y)=\sum_{|Y|=r} \Omega_{Y}^{(g, p)}(\lambda, y)
$$

Formula (1.12) follows by a straightforward computation.

\subsection{Double refinement}

Physical arguments [15] present compelling evidence for the existence of a doubly refined BPS counting function, which is graded by $U(1)_{R}$ charge in 
addition to spin quantum number. In this section it is conjectured that the doubly refined partition function of asymptotic ADHM invariants is obtained again from the equivariant instanton sum (3.4) by a different specialization of the equivariant parameters. Namely, for $r \in \mathbb{Z}_{\geq 1}$, any Young diagram $Y$ with $|Y|=r$, and any $p \in \mathbb{Z}$ let

$$
\Omega_{(Y, \emptyset)}^{(g, p)}\left(\lambda, u^{1 / 2}, v^{1 / 2}\right)=u^{(g+1)|\mu|} v^{(g-1)|\mu|} \mathcal{Z}_{(Y, \emptyset)}^{(g, p)}\left(\lambda^{-1}(u v)^{1 / 2}, \lambda(u v)^{1 / 2}, u^{-1}\right)^{(0)} .
$$

The generating function of doubly refined asymptotic ADHM invariants is then conjectured to be

$$
\mathcal{Z}_{+\infty}(\mathcal{X}, r ; \lambda, u, v)=\sum_{|Y|=r} \Omega_{Y}^{(g, p)}\left(\lambda, u^{1 / 2}, v^{1 / 2}\right)
$$

A straightforward computation yields formula (1.15). In conjunction with the doubly refined wallcrossing conjecture (1.3), the above formula will be shown to yield correct results for the Hodge polynomial of the Hitchin moduli space in many examples recorded in Appendix B.

\subsection{Localization interpretation for $r=2$}

Suppose the conditions of Section 2.2 are satisfied, that is $p \geq 0$, and $M_{1}=$ $\mathcal{O}_{X}, M_{2}=K_{X}^{-1}$ if $p=0$. The goal of this section is to discuss the geometric interpretation of Conjecture 1.5 for $r=1,2$. The main observation is that in these cases, Equation (1.11) can be interpreted as a sum of contributions of torus fixed loci in the moduli space $\mathfrak{M}_{+\infty}^{\mathrm{ss}}(\mathcal{X}, r, e)$. However, a rigorous geometric computation would require a localization theorem for the refined Donaldson-Thomas invariants defined in [42], which has not been formulated and proven so far.

First let $r=1$. The moduli stack of $\delta$-semistable ADHM sheaves of type $(1, e)$ on $X$ with $\delta>0$ and $e \geq 0$ is a $\mathbb{C}^{\times}$-gerbe over the smooth variety

$$
S^{e}(X) \times H^{0}\left(X, M_{1}^{-1}\right) \times H^{0}\left(X, M_{2}^{-1}\right) .
$$

A $\mathbb{C}$-valued point of $\mathfrak{M}_{\delta}^{\text {ss }}(\mathcal{X}, 1, e)$ is an ADHM sheaf of the form $\left(E, \Phi_{1}, \Phi_{2}, 0\right.$, $\psi$ ) where $E$ is a degree $e$ line bundle on $X, \Phi_{1} \in \operatorname{Hom}_{X}\left(E \otimes_{X} M_{1}, E\right) \simeq$ $H^{0}\left(X, M_{1}^{-1}\right), \Phi_{2} \in \operatorname{Hom}_{X}\left(E \otimes_{X} M_{2}, E\right) \simeq H^{0}\left(X, M_{2}^{-1}\right)$ and $\psi \in H^{0}(X, E)$. The $\delta$-stability condition, $\delta>0$ is equivalent to $\psi$ not identically zero. Obviously, the moduli stack is empty if $e<0$.

The fixed point conditions require $\Phi_{1}=0, \Phi_{2}=0$. Therefore the torus fixed locus is a $\mathbb{C}^{\times}$-gerbe over the symmetric product $S^{e}(X)$. 
Conjecture 1.5 and Equation (1.12) yield

$$
\mathcal{Z}_{+\infty}(\mathcal{X}, 1 ; \lambda, y)=(-1)^{p} y^{1-g} \frac{(1-\lambda)^{2 g}}{(1-\lambda y)\left(1-\lambda y^{-1}\right)}
$$

Now recall Macdonald's formula

$$
\sum_{n \geq 0} P_{z}\left(S^{n}(X)\right) x^{n}=\frac{(1-x z)^{2 g}}{(1-x)\left(1-x z^{2}\right)}
$$

for the generating function of Poincaré polynomials of symmetric products of $X$. Then Equations (3.17) and (3.18) imply

$$
\mathcal{Z}_{+\infty}(\mathcal{X}, 1 ; \lambda, y)=(-1)^{p} \sum_{e \geq 0} \lambda^{e} y^{1-g-e} P_{y}\left(S^{e}(X)\right)
$$

for all $e \in \mathbb{Z}_{\geq 0}$.

Next let $r=2$. Property (B.2) implies that the moduli space $\mathfrak{M}_{+\infty}^{\mathrm{ss}}(\mathcal{X}$, $r, e)$ is empty unless $e \geq 2-2 g$. Assuming this to be the case, a straightforward analysis shows that the components of the torus fixed locus are of two types. The ADHM sheaves corresponding to the $\mathbb{C}$-valued fixed points are presented as follows:

(i) $E \simeq E_{-1} \oplus E_{0}, \Phi_{2}=0, \operatorname{Im}(\psi) \subseteq E_{0}$ and

$$
\Phi_{1}=\left[\begin{array}{ll}
0 & \varphi \\
0 & 0
\end{array}\right]
$$

with $\varphi: E_{0} \otimes_{X} M_{1} \rightarrow E_{-1}$ a nontrivial morphism of line bundles. Components of this type are isomorphic to $\mathbb{C}^{\times}$-gerbes over the smooth varieties

$$
S^{e_{0}}(X) \times S^{e_{-1}-e_{0}-p}(X)
$$

where $0 \leq e_{0} \leq e_{-1}-p$ and $e_{0}+e_{-1}=e$.

(ii) $E \simeq E_{0} \oplus E_{1}, \Phi_{1}=0, \operatorname{Im}(\psi) \subseteq E_{0}$ and

$$
\Phi_{2}=\left[\begin{array}{ll}
0 & 0 \\
\varphi & 0
\end{array}\right]
$$

with $\varphi: E_{0} \otimes_{X} M_{2} \rightarrow E_{1}$ a nontrivial morphism of line bundles. Components of this type are isomorphic to $\mathbb{C}^{\times}$-gerbes over the smooth 
varieties

$$
S^{e_{0}}(X) \times S^{e_{1}-e_{0}+2 g-2+p}(X)
$$

where $0 \leq e_{0} \leq e_{1}+2 g-2+p$ and $e_{0}+e_{1}=e$.

Note that in both cases, the moduli stack of asymptotically stable ADHM sheaves is not smooth along the fixed loci, although the fixed loci are smooth.

Conjecture 1.5 and Equation (1.12) yield

$$
\begin{aligned}
\mathcal{Z}_{+\infty}(\mathcal{X}, 2 ; \lambda, y) & =\Omega_{\square}^{(g, p)}(\lambda, y)+\Omega_{\boxminus}^{(g, p)}(\lambda, y) \\
\Omega_{\boxminus}^{(g, p)}(\lambda, y) & =\left(\lambda^{-1} y\right)^{-p} y^{2-2 g} \frac{\left(1-\lambda^{2} y^{-1}\right)^{2 g}(1-\lambda)^{2 g}}{\left(1-\lambda^{2}\right)\left(1-\lambda^{2} y^{-2}\right)(1-\lambda y)\left(1-\lambda y^{-1}\right)} \\
& \Omega_{\square}^{(g, p)}(\lambda, y)=(\lambda y)^{-p} y^{4-4 g} \lambda^{2-2 g} \frac{\left(1-\lambda^{2} y\right)^{2 g}(1-\lambda)^{2 g}}{\left(1-\lambda^{2}\right)\left(1-\lambda^{2} y^{2}\right)(1-\lambda y)\left(1-\lambda y^{-1}\right)} .
\end{aligned}
$$

A straightforward computation using Equation (3.18) yields

$$
\begin{aligned}
& \Omega_{\boxminus}^{(g, p)}(\lambda, y)=\sum_{e \geq p} \lambda^{e} \sum_{\substack{e_{0}+e_{-1}=e \\
0 \leq e_{0} \leq e_{-1}-p}} y^{2-2 g-p-e_{0}} y^{-e_{0}} P_{y}\left(S^{e_{0}}(X)\right) \\
& y^{-e_{-1}+e_{0}+p} P_{y}\left(S^{e_{-1}-e_{0}-p}(X)\right) \\
& \Omega_{\square}^{(g, p)}(\lambda, y)=\sum_{e \geq 2-2 g-p} \lambda^{e} \sum_{\substack{e_{0}+e_{1}=e \\
0 \leq e_{0} \leq e_{1}+2 g-2+p}} y^{e_{0}-p} y^{-e_{0}} P_{y}\left(S^{e_{0}}(X)\right) \\
& y^{-e_{1}+e_{0}-2 g+2-p} P_{y}\left(S^{e_{1}-e_{0}+2 g-2+p}(X)\right) \text {. }
\end{aligned}
$$

Given the explicit description of the fixed loci, Equations (3.17) and (3.19)(3.21) clearly suggest an equivariant localization theorem for refined ADHM invariants. Such a formula would presumably allow a rigorous computation of the polynomial weights assigned to each component of the fixed locus.

For future reference, let us record the expressions $\Omega_{Y}^{(p)}(\lambda, y)$ for $|Y|=3$.

$$
\begin{aligned}
& \Omega_{\boxminus}^{(g, p)}(\lambda, y)=(-1)^{p}\left(\lambda^{3} y^{-3}\right)^{p} y^{3-3 g} \frac{(1-\lambda)^{2 g}\left(1-\lambda^{2} y^{-1}\right)^{2 g}\left(1-\lambda^{3} y^{-2}\right)^{2 g}}{(1-\lambda y)\left(1-\lambda y^{-1}\right)\left(1-\lambda^{2} y^{-2}\right)\left(1-\lambda^{2}\right)} \\
& \Omega_{\boxminus}^{(g, p)}(\lambda, y)=(-1)^{p} y^{2 p} y^{5-5 g} \lambda^{2-2 g} \frac{\left(1-\lambda^{3} y^{-3}\right)\left(1-\lambda^{3} y^{-1}\right)}{(1-\lambda y)^{2}\left(1-\lambda y^{-1}\right)^{2}\left(1-\lambda^{3} y\right)\left(1-\lambda^{3} y^{-1}\right)}
\end{aligned}
$$




$$
\begin{aligned}
\Omega_{\square}^{(g, p)}(\lambda, y)= & (-1)^{p}\left(\lambda^{-3} y^{-3}\right)^{p} y^{9-9 g} \lambda^{6-6 g} \\
& \times \frac{(1-\lambda)^{2 g}\left(1-\lambda^{2} y\right)^{2 g}\left(1-\lambda^{3} y^{2}\right)^{2 g}}{(1-\lambda y)\left(1-\lambda y^{-1}\right)\left(1-\lambda^{2} y^{2}\right)\left(1-\lambda^{2}\right)\left(1-\lambda^{3} y^{3}\right)\left(1-\lambda^{3} y\right)}
\end{aligned}
$$

\section{Examples, comparison with existing results}

This section will present several concrete results for Poincaré polynomials of moduli spaces of Hitchin pairs obtained from the recursion relation (1.8) and Conjecture 1.5. In all cases considered below, these results are identical to the computations of Hitchin [29] and Gothen [24], as well as the conjecture of Hausel and Rodriguez-Villegas [27,28], which are briefly reviewed in Appendix A. In addition, entirely analogous computations have been done for the Hodge polynomial of moduli spaces of pairs, employing the doubly refined version of the recursion formula and Conjecture 1.6. The results are presented in Appendix B. Again, all cases considered there are in agreement with the results of [24,27-29].

In order to simplify the formulas set $\widetilde{A}_{+\infty}(r, e)(y)=(-1)^{r p} A_{+\infty}(r, e)(y)$, $\widetilde{H}(r, e)(y)=(-1)^{e-r(g-1-p)} H(r, e)(y)$ for all $(r, e) \in \mathbb{Z}_{\geq 1} \times \mathbb{Z}$. Then Equation (1.8) becomes

$$
\begin{aligned}
& {[e-r(g-1)]_{y} \widetilde{H}(\gamma)(y)} \\
& =\widetilde{A}_{+\infty}(\gamma)(y)-\widetilde{A}_{+\infty}(\widetilde{\gamma})(y)+\sum_{l \geq 2} \frac{(-1)^{l-1}}{(l-1) !} \sum_{\begin{array}{c}
\gamma_{1}, \ldots, \gamma_{l} \in \mathbb{Z}_{\geq 1} \times \mathbb{Z} \\
\gamma_{1}+\cdots+\gamma_{l}=\gamma
\end{array}} \\
& \mu_{0}(r) \leq \mu(\gamma)<\mu\left(\gamma_{i}\right), 2 \leq i \leq l, \\
& \times \widetilde{A}_{+\infty}\left(\gamma_{1}\right)(y) \prod_{i=2}^{l}\left[e_{i}-r_{i}(g-1)\right]_{y} \widetilde{H}\left(r_{i}, e_{i}\right)(y)-\sum_{l \geq 2} \frac{(-1)^{l-1}}{(l-1) !} \\
& \times \quad \sum_{\gamma_{1}, \ldots, \gamma_{l} \in \mathbb{Z}_{\geq 1} \times \mathbb{Z}} \widetilde{A}_{+\infty}\left(\gamma_{1}\right)(y) \prod_{i=2}^{l}\left[e_{i}-r_{i}(g-1)\right]_{y} \widetilde{H}\left(r_{i}, e_{i}\right)(y) \\
& \begin{array}{c}
\mu_{0}(r) \leq \mu(\widetilde{\gamma}) \leq \mu\left(\gamma_{i}\right), 2 \leq i \leq l \\
\mu_{0}(r) \leq \mu\left(\gamma_{1}\right)
\end{array} \\
& -\sum_{l \geq 2} \frac{1}{l !} \sum_{\substack{\gamma_{1}, \ldots, \gamma_{l} \in \mathbb{Z}_{\geq 1} \times \mathbb{Z} \\
\gamma_{1}+\cdots+\gamma_{l}=\gamma \\
\mu(\gamma)=\mu\left(\gamma_{i}\right), 1 \leq i \leq l}} \prod_{i=1}^{l}\left[e_{i}-r_{i}(g-1)\right]_{y} \widetilde{H}\left(r_{i}, e_{i}\right)(y)
\end{aligned}
$$


where $\mu_{0}(r)=-(r-1)(2 g-2+p)$, and the sum on the right-hand side of Equation (1.8) is finite.

\subsection{Rank $r=1$}

There are no positive critical parameters of type $(1, e)$ for any $e \in \mathbb{Z}_{\geq 0}$ The wallcrossing formula (1.4) at $\delta_{c}=0$ reads

$$
\widetilde{A}_{+\infty}(1, e)-\widetilde{A}_{+\infty}(1,-e+2(g-1))=[e-g+1]_{y} \widetilde{H}(1, e) .
$$

Expanding the right-hand side of Equation (3.19) in powers of $\lambda$ yields

$$
\widetilde{A}_{+\infty}(1, e)=y^{1-g} \sum_{\substack{0 \leq k \leq 2 g \\ m, l \geq 0, k+l+m=e}}(2 g, k)(-1)^{k} y^{l-m}
$$

for any $e \geq 0$, where $(2 g, k)=\frac{(2 g) !}{k !(2 g-k) !}$ are binomial coefficients. A series of elementary manipulations further yield

$$
\begin{aligned}
\widetilde{A}_{+\infty}(1, e) & =y^{1-g} \sum_{\substack{0 \leq k \leq 2 g \\
l \geq 0, l+k \leq e}}(2 g, k)(-1)^{k} y^{2 l+k-e} \\
& =y^{1-g} \sum_{\substack{0 \leq k \leq 2 g \\
l \geq 0, l+k \leq e}}(2 g, k)(-1)^{k} y^{k-e} \frac{1-y^{2 e-2 k+2}}{1-y^{2}} \\
& =\frac{y^{1-g}}{1-y^{2}} \sum_{\substack{0 \leq k \leq 2 g \\
k \leq e}}(2 g, k)(-1)^{k}\left(y^{k-e}-y^{e-k+2}\right)
\end{aligned}
$$

for any $e \geq 0$. In order to compute the left-hand side of Equation (4.2), it is convenient to consider three cases.

(a) $0 \leq e \leq 2 g-2$. Then

$$
\begin{aligned}
\widetilde{A}_{+\infty}(1, e)-\widetilde{A}_{+\infty}(1,-e+2(g-1)) & \\
= & \frac{y^{1-g}}{1-y^{2}}\left[\sum_{k=0}^{e}(2 g, k)(-1)^{k} y^{k-e}+\sum_{k=0}^{2 g-2-e}(2 g, k)(-1)^{k} y^{2 g-2-e-k}\right] \\
& -\frac{y^{1-g}}{1-y^{2}}\left[\sum_{k=0}^{e}(2 g, k)(-1)^{k} y^{e-k+2}+\sum_{k=0}^{2 g-2-e}(2 g, k)(-1)^{k} y^{k+e-2 g+2}\right]
\end{aligned}
$$




$$
\begin{aligned}
= & \frac{y^{1-g}}{1-y^{2}} y^{-e}\left[\sum_{k=0}^{e}(2 g, k)(-1)^{k} y^{k}+\sum_{k=e+2}^{2 g-2}(2 g, k)(-1)^{k} y^{k}\right] \\
& -\frac{y^{1-g}}{1-y^{2}} y^{e+2}\left[\sum_{k=0}^{e}(2 g, k)(-1)^{k} y^{-k}+\sum_{k=e+2}^{2 g-2}(2 g, k)(-1)^{k} y^{-k}\right] \\
= & -\frac{y^{1-g}}{1-y^{2}}\left[y^{e+2}\left(1-y^{-1}\right)^{2 g}-y^{-e}(1-y)^{2 g}\right] \\
= & \frac{y^{e-g+1}-y^{-e+g-1}}{y-y^{-1}} \frac{(1-y)^{2 g}}{y^{2 g-1}} .
\end{aligned}
$$

(b) $e=2 g-1$. Then $\widetilde{A}_{+\infty}(-e+2 g-2)=0$ and

$$
\begin{aligned}
\widetilde{A}_{+\infty}(1,2 g-1) & =\frac{y^{1-g}}{1-y^{2}} \sum_{k=0}^{2 g-1}(2 g, k)(-1)^{k}\left(y^{k-2 g+1}-y^{2 g-k+1}\right) \\
& =\frac{y^{1-g}}{1-y^{2}}\left[y^{1-2 g}(1-y)^{2 g}-y^{2 g+1}\left(1-y^{-1}\right)^{2 g}\right] \\
& =\frac{y^{g}-y^{-g}}{y-y^{-1}} \frac{(1-y)^{2 g}}{y^{2 g-1}} .
\end{aligned}
$$

(c) $e \geq 2 g$. Then $\widetilde{A}_{+\infty}(-e+2 g-2)=0$ and a similar computation yields

$$
\widetilde{A}_{+\infty}(1, e)=\frac{y^{e-g+1}-y^{-e+g-1}}{y-y^{-1}} \frac{(1-y)^{2 g}}{y^{2 g-1}} .
$$

In conclusion,

$$
\widetilde{H}(1, e)(y)=\frac{(1-y)^{2 g}}{y^{2 g-1}}
$$

for all $e \geq 0$, hence also for all $e \in \mathbb{Z}$.

The moduli space of rank one semistable Hitchin pairs of any degree $e \in \mathbb{Z}$ is isomorphic to

$$
H^{0}\left(X, M_{1}^{-1}\right) \times H^{0}\left(X, M_{2}^{-1}\right) \times J_{e}(X)
$$

where $J_{e}(X)$ is the degree $e$ Jacobian of $X$. Obviously formula (4.3) can be rewritten as

$$
\widetilde{H}(1, e)(y)=y^{1-2 g} P_{y}\left(J_{e}(X)\right)
$$

for any $e \in \mathbb{Z}$. 


\subsection{Rank $r=2$}

According to property (B.2) in Section 2.1, all invariants $A_{\delta}(1, e)(y)$ are zero for $e<0$. It will be convenient to distinguish two cases, depending on the parity of $e$. By convention, any sum in the following formulas is zero if the lower summation bound exceeds the upper summation bound.

(a) $e=2 n, n \in \mathbb{Z}$. Then Equation (4.1) reduces to

$$
\begin{aligned}
& {[2 n-2 g+2]_{y} \widetilde{H}(2,2 n)(y)=\widetilde{A}_{+\infty}(2,2 n)-\widetilde{A}_{+\infty}(2,-2 n+4 g-4)} \\
& \quad-\sum_{e_{1}=0}^{n-1} \widetilde{A}_{+\infty}\left(1, e_{1}\right)(y)\left[2 n-e_{1}-g+1\right]_{y} \widetilde{H}\left(1,2 n-e_{1}\right)(y) \\
& \quad+\sum_{e_{1}=0}^{2 g-2-n} \widetilde{A}_{+\infty}\left(1, e_{1}\right)(y)\left[3 g-3-2 n-e_{1}\right]_{y} \widetilde{H}(1,4 g-4-2 n)(y) \\
& \quad-\frac{1}{2}[n-g+1]_{y}^{2} \widetilde{H}(1, n)(y)^{2} .
\end{aligned}
$$

(b) $e=2 n+1, n \in \mathbb{Z}$. Then Equation (1.8) reduces to

$$
\begin{aligned}
& {[2 n-2 g+3]_{y} \widetilde{H}(2,2 n+1)(y)=\widetilde{A}_{+\infty}(2,2 n+1)-\widetilde{A}_{+\infty}(2,4 g-5-2 n)} \\
& \quad-\sum_{e_{1}=0}^{n} \widetilde{A}_{+\infty}\left(1, e_{1}\right)(y)\left[2 n-e_{1}-g+2\right]_{y} \widetilde{H}\left(1,2 n+1-e_{1}\right)(y) \\
& \quad+\sum_{e_{1}=0}^{2 g-3-n} \widetilde{A}_{+\infty}\left(1, e_{1}\right)(y)\left[3 g-4-2 n-e_{1}\right]_{y} H\left(1,4 g-4-2 n-e_{1}\right)(y) .
\end{aligned}
$$

Some concrete results are recorded below. $\widetilde{H}^{(p)}(r, e)$ denotes the refined Higgs invariant of type $(r, e)$ with coefficient bundles $\left(M_{1}, M_{2}\right)$ of degrees $(p, 2-2 g-p), p \geq 0$. Under the current assumptions, $M_{1} \simeq$ $\mathcal{O}_{X}$ if $p=0$.

$$
g=2
$$

$$
\begin{gathered}
\widetilde{H}^{(0)}(2,1)(y)=\frac{(1-y)^{4}\left(1+y^{2}\right)\left(1-4 y^{3}+2 y^{4}\right)}{y^{9}} \\
\widetilde{H}^{(0)}(2,0)(y)=\frac{(1-y)^{4}\left(2+4 y^{2}-8 y^{3}+7 y^{4}-12 y^{5}+14 y^{6}-4 y^{7}+5 y^{8}\right)}{2 y^{9}\left(1+y^{2}\right)} \\
\widetilde{H}^{(1)}(2,1)(y)=\frac{(1-y)^{4}\left(2 y^{8}-4 y^{7}+8 y^{6}-4 y^{5}+2 y^{4}-4 y^{3}+y^{2}+1\right)}{y^{11}}
\end{gathered}
$$




$$
\begin{aligned}
& \widetilde{H}^{(1)}(2,0)(y)=\frac{(1-y)^{4}\left(3 y^{10}-12 y^{9}+14 y^{8}-20 y^{7}+19 y^{6}-16 y^{5}\right.}{\left.+6 y^{4}-8 y^{3}+4 y^{2}+2\right)} \\
& \widetilde{H}^{(2)}(2,1)(y)=\frac{(1-y)^{4}\left(y^{2}+1\right)\left(2 y^{8}-8 y^{7}+6 y^{6}+2 y^{4}-4 y^{3}+1\right)}{y^{13}} \\
& \widetilde{H}^{(2)}(2,0)(y)=\frac{(1-y)^{4}\left(5 y^{12}-12 y^{11}+26 y^{10}-28 y^{9}+33 y^{8}-24 y^{7}\right.}{\left.+20 y^{6}-16 y^{5}+6 y^{4}-8 y^{3}+4 y^{2}+2\right)} \\
& 2 y^{13}\left(1+y^{2}\right)
\end{aligned}
$$$$
\begin{aligned}
\widetilde{H}^{(0)}(2,1)(y)= & \frac{(1-y)^{6}}{y^{17}}\left(1+y^{2}-6 y^{3}+2 y^{4}-6 y^{5}+17 y^{6}-12 y^{7}+18 y^{8}\right. \\
& \left.-32 y^{9}+18 y^{10}-12 y^{11}+3 y^{12}\right)
\end{aligned}
$$$$
\widetilde{H}^{(0)}(2,0)(y)=\frac{(1-y)^{6}}{2 y^{17}\left(1+y^{2}\right)}\left(2+4 y^{2}-12 y^{3}+6 y^{4}-24 y^{5}+38 y^{6}-36 y^{7}\right.
$$$$
\left.+71 y^{8}-82 y^{9}+87 y^{10}-68 y^{11}+57 y^{12}-18 y^{13}+7 y^{14}\right)
$$$$
(1-y)^{6}\left(y^{2}+1\right)\left(3 y^{12}-12 y^{11}+30 y^{10}\right.
$$$$
\widetilde{H}^{(1)}(2,1)(y)=\frac{\left.-20 y^{9}+3 y^{8}-12 y^{7}+15 y^{6}+2 y^{4}-6 y^{3}+1\right)}{y^{19}}
$$$$
\widetilde{H}^{(1)}(2,0)(y)=\frac{(1-y)^{6}}{2 y^{19}\left(1+y^{2}\right)}\left(5 y^{16}-30 y^{15}+57 y^{14}-108 y^{13}+117 y^{12}-134 y^{11}\right.
$$$$
+101 y^{10}-88 y^{9}+70 y^{8}-36 y^{7}+38 y^{6}-24 y^{5}
$$$$
\left.+6 y^{4}-12 y^{3}+4 y^{2}+2\right)
$$$$
\widetilde{H}^{(2)}(2,1)(y)=\frac{(1-y)^{6}}{y^{21}}\left(3 y^{16}-18 y^{15}+33 y^{14}-52 y^{13}+48 y^{12}-38 y^{11}+33 y^{10}\right.
$$$$
\left.-32 y^{9}+18 y^{8}-12 y^{7}+17 y^{6}-6 y^{5}+2 y^{4}-6 y^{3}+y^{2}+1\right)
$$$$
\widetilde{H}^{(2)}(2,0)(y)=\frac{(1-y)^{6}}{2 y^{21}\left(1+y^{2}\right)}\left(7 y^{18}-30 y^{17}+87 y^{16}-120 y^{15}+177 y^{14}-174 y^{13}\right.
$$$$
+163 y^{12}-140 y^{11}+102 y^{10}-88 y^{9}+70 y^{8}-36 y^{7}
$$$$
\left.+38 y^{6}-24 y^{5}+6 y^{4}-12 y^{3}+4 y^{2}+2\right) \text {. }
$$

$g=4$

$$
\begin{aligned}
\widetilde{H}^{(0)}(2,1)(y)= & \frac{(1-y)^{8}}{y^{25}}\left(1+y^{2}\right)\left(1-8 y^{3}+2 y^{4}+28 y^{6}-16 y^{7}+3 y^{8}-56 y^{9}\right. \\
& \left.+56 y^{10}-24 y^{11}+74 y^{12}-112 y^{13}+56 y^{14}-24 y^{15}+4 y^{16}\right)
\end{aligned}
$$




$$
\begin{aligned}
\widetilde{H}^{(0)}(2,0)(y)= & \frac{(1-y)^{8}}{2 y^{25}\left(1+y^{2}\right)}\left(2+4 y^{2}-16 y^{3}+6 y^{4}-32 y^{5}+64 y^{6}-48 y^{7}\right. \\
& +122 y^{8}-176 y^{9}+180 y^{10}-304 y^{11}+379 y^{12}-424 y^{13}+548 y^{14} \\
& \left.-488 y^{15}+450 y^{16}-264 y^{17}+156 y^{18}-40 y^{19}+9 y^{20}\right) . \\
g=5 & \\
\widetilde{H}^{(0)}(2,1)(y)= & \frac{(1-y)^{10}}{y^{33}}\left(1+y^{2}-10 y^{3}+2 y^{4}-10 y^{5}+47 y^{6}-20 y^{7}+48 y^{8}\right. \\
& -140 y^{9}+93 y^{10}-150 y^{11}+304 y^{12}-270 y^{13}+349 y^{14} \\
& -532 y^{15}+560 y^{16}-652 y^{17}+770 y^{18}-784 y^{19} \\
& \left.+560 y^{20}-400 y^{21}+140 y^{22}-40 y^{23}+5 y^{24}\right) .
\end{aligned}
$$

In all the above cases, similar computations also show that the invariants $\widetilde{H}(2, e)$ depend only on the parity of $e \in \mathbb{Z}$. Note also that for even $e$ the rank two refined Higgs invariants are rational functions of $y$ rather than polynomials in $y^{-1}, y$. By analogy with the theory of generalized DonaldsonThomas invariants [38], this reflects the fact that in this case the moduli stack $\mathfrak{H i g} \mathfrak{i g} \mathfrak{s}^{\mathrm{ss}}(\mathcal{X}, 2, e)$ contains strictly semistable $\mathbb{C}$-valued points.

\subsection{Rank $r=3$}

According to property (B.2) in Section 2.1, all invariants $A_{\delta}(2, e)(y)$ are zero for $e<2-2 g-p$. Suppose $e=3 n+1, n \in \mathbb{Z}$. Then Equation (4.1) reduces to

$$
\begin{aligned}
& {[3 n-3 g+4]_{y} \widetilde{H}(3,3 n+1)=\widetilde{A}_{+\infty}(3,3 n+1)(y)-\widetilde{A}_{+\infty}(3,-3 n+6 g-7)(y)} \\
& \quad-\sum_{e_{1}=2-2 g-p}^{2 n} \widetilde{A}_{+\infty}\left(2, e_{1}\right)\left[3 n+2-g-e_{1}\right]_{y} \widetilde{H}\left(1,3 n+1-e_{1}\right)(y) \\
& \quad-\sum_{e_{1}=0}^{n} \widetilde{A}_{+\infty}\left(1, e_{1}\right)\left[3 n+3-2 g-e_{1}\right]_{y} \widetilde{H}\left(2,3 n+1-e_{1}\right)(y) \\
& \quad+\frac{1}{2} \sum_{e_{1}=0}^{n-1} \sum_{e_{2}=n+1}^{2 n-e_{1}} \widetilde{A}_{+\infty}\left(1, e_{1}\right)\left[e_{2}-g+1\right]_{y}\left[3 n+2-g-e_{1}-e_{2}\right]_{y} \\
& \quad \times \widetilde{H}\left(1,3 n+1-e_{1}-e_{2}\right)(y)^{2}+\sum_{e_{1}=2-2 g-p}^{4 g-2 n-5} \widetilde{A}_{+\infty}\left(2, e_{1}\right)\left[5 g-6-3 n-e_{1}\right]_{y}
\end{aligned}
$$




$$
\begin{aligned}
& \times \widetilde{H}\left(1,6 g-7-3 n-e_{1}\right)(y)+\sum_{e_{1}=0}^{2 g-n-3} \widetilde{A}_{+\infty}\left(1, e_{1}\right)\left[4 g-5-3 n-e_{1}\right]_{y} \\
& \times \widetilde{H}\left(2,6 g-7-3 n-e_{1}\right)(y)-\frac{1}{2} \sum_{e_{1}=0}^{2 g-3-n} \sum_{e_{2}=2 g-2-n}^{4 g-2 n-5-e_{1}} \widetilde{A}_{+\infty}\left(1, e_{1}\right)\left[e_{2}-g+1\right]_{y} \\
& \times\left[5 g-6-3 n-e_{1}-e_{2}\right]_{y} \widetilde{H}\left(1,6 g-7-3 n-e_{1}-e_{2}\right)(y)^{2} .
\end{aligned}
$$

Again, some concrete results are recorded below.

$$
\begin{aligned}
& g=2 \\
& \widetilde{H}^{(0)}(3,1)(y)=\frac{(1-y)^{4}}{y^{19}}\left(1+y^{2}-4 y^{3}+3 y^{4}-8 y^{5}+10 y^{6}-16 y^{7}+29 y^{8}\right. \\
& -32 y^{9}+48 y^{10}-64 y^{11}+67 y^{12}-68 y^{13} \\
& \left.+48 y^{14}-24 y^{15}+6 y^{16}\right) \\
& \widetilde{H}^{(1)}(3,1)(y)=\frac{(1-y)^{4}}{y^{25}}\left(6 y^{22}-36 y^{21}+96 y^{20}-168 y^{19}+207 y^{18}-216 y^{17}\right. \\
& +210 y^{16}-184 y^{15}+149 y^{14}-120 y^{13}+92 y^{12}-72 y^{11}+49 y^{10} \\
& \left.-32 y^{9}+29 y^{8}-16 y^{7}+10 y^{6}-8 y^{5}+3 y^{4}-4 y^{3}+y^{2}+1\right) \\
& \widetilde{H}^{(2)}(3,1)(y)=\frac{(1-y)^{4}}{y^{31}}\left(10 y^{28}-64 y^{27}+184 y^{26}-344 y^{25}+477 y^{24}-560 y^{23}\right. \\
& +583 y^{22}-560 y^{21}+522 y^{20}-464 y^{19}+386 y^{18}-320 y^{17} \\
& +267 y^{16}-208 y^{15}+158 y^{14}-124 y^{13}+93 y^{12}-72 y^{11}+49 y^{10} \\
& \left.-32 y^{9}+29 y^{8}-16 y^{7}+10 y^{6}-8 y^{5}+3 y^{4}-4 y^{3}+y^{2}+1\right) \text {. } \\
& g=3 \\
& \widetilde{H}^{(0)}(3,1)(y)=\frac{(1-y)^{6}}{y^{37}}\left(15 y^{32}-120 y^{31}+480 y^{30}-1260 y^{29}+2355 y^{28}\right. \\
& -3486 y^{27}+4189 y^{26}-4416 y^{25}+4315 y^{24}-3922 y^{23} \\
& +3399 y^{22}+2309 y^{20}-1872 y^{19}+1433 y^{18}-1072 y^{17} \\
& +861 y^{16}-604 y^{15}-2860 y^{21}+446 y^{14}-336 y^{13}+212 y^{12} \\
& -176 y^{11}+105 y^{10}-62 y^{9}+58 y^{8}-24 y^{7}+19 y^{6} \\
& \left.-12 y^{5}+3 y^{4}-6 y^{3}+y^{2}+1\right) \\
& \widetilde{H}^{(1)}(3,1)(y)=\frac{(1-y)^{6}}{y^{43}}\left(15 y^{38}-150 y^{37}+690 y^{36}-2010 y^{35}+4110 y^{34}\right. \\
& -6542 y^{33}+8598 y^{32}-9930 y^{31}+10427 y^{30}-10254 y^{29}
\end{aligned}
$$




$$
\begin{aligned}
& +9672 y^{28}-8800 y^{27}+7705 y^{26}-6600 y^{25}+5598 y^{24} \\
& -4600 y^{23}+3723 y^{22}-3006 y^{21}+2363 y^{20}-1884 y^{19} \\
& +1434 y^{18}-1072 y^{17}+861 y^{16}-604 y^{15}+446 y^{14}-336 y^{13} \\
& +212 y^{12}-176 y^{11}+105 y^{10}-62 y^{9}+58 y^{8} \\
& \left.-24 y^{7}+19 y^{6}-12 y^{5}+3 y^{4}-6 y^{3}+y^{2}+1\right) \\
\widetilde{H}^{(2)}(3,1)(y)= & \frac{(1-y)^{6}}{} y^{49}\left(21 y^{44}-216 y^{43}+1026 y^{42}-3090 y^{41}+6621 y^{40}\right. \\
& -11094 y^{39}+15375 y^{38}-18672 y^{37}+20712 y^{36}-21584 y^{35} \\
& +21450 y^{34}-20552 y^{33}+19178 y^{32}-17460 y^{31}+15503 y^{30} \\
& -13546 y^{29}+11706 y^{28}-9952 y^{27}+8316 y^{26}-6912 y^{25} \\
& +5736 y^{24}-4650 y^{23}+3741 y^{22}-3012 y^{21}+2364 y^{20} \\
& -1884 y^{19}+1434 y^{18}-1072 y^{17}+861 y^{16}-604 y^{15}+446 y^{14} \\
& -336 y^{13}+212 y^{12}-176 y^{11}+105 y^{10}-62 y^{9}+58 y^{8} \\
& \left.-24 y^{7}+19 y^{6}-12 y^{5}+3 y^{4}-6 y^{3}+y^{2}+1\right) .
\end{aligned}
$$

$g=4$

$$
\begin{aligned}
\widetilde{H}^{(0)}(3,1)(y)= & \frac{(1-y)^{8}}{y^{55}}\left(28 y^{48}-336 y^{47}+2016 y^{46}-7896 y^{45}+22218 y^{44}\right. \\
& -48328 y^{43}+84084 y^{42}-122616 y^{41}+155235 y^{40} \\
& -176912 y^{39}+186320 y^{38}-185408 y^{37}+176976 y^{36}-163656 y^{35} \\
& +146930 y^{34}-128936 y^{33}+111544 y^{32}-94416 y^{31}+78918 y^{30} \\
& -65392 y^{29}+53178 y^{28}-43392 y^{27}+34620 y^{26}-27288 y^{25} \\
& +21936 y^{24}-16728 y^{23}+13005 y^{22}-10064 y^{21}+7290 y^{20} \\
& -5760 y^{19}+4077 y^{18}-2880 y^{17}+2278 y^{16}-1416 y^{15} \\
& +1071 y^{14}-744 y^{13}+416 y^{12}-368 y^{11}+185 y^{10}-112 y^{9} \\
& \left.+99 y^{8}-32 y^{7}+32 y^{6}-16 y^{5}+3 y^{4}-8 y^{3}+y^{2}+1\right) .
\end{aligned}
$$

In addition similar computations show that $\widetilde{H}^{(p)}(3,2)(y)=\widetilde{H}^{(p)}(3,1)(y)$ in all above examples.

\section{Acknowledgments}

We are very grateful to Ugo Bruzzo, Ron Donagi, Daniel Jafferis, Yunfeng Jiang, Dominic Joyce, Greg Moore, Artan Sheshmani, Balasz Szendroi, Chris 
Woodward, and especially Tamas Hausel, Ludmil Katzarkov, Sergey Mozgovoy, Tony Pantev and Fernando Rodriguez-Villegas for their interest in this work and many helpful discussions. We owe special thanks to Sergey Mozgovoy for sending us his paper [45] before publication. D.E.D. would also like to thank the organizers of VBAC 2009 Berlin for an excellent mathematical atmosphere which prompted the research reported here. The work of W.Y.C. is supported by DOE grant DE-FG02-96ER40959. The work of D.E.D. was partially supported by NSF grant PHY-0854757-2009.

\section{Appendix A. Existing results}

This section is a summary of existing results and conjectures on the cohomology of moduli spaces of Hitchin pairs. The localization computations of Hitchin [29] and Gothen [24] as well as the conjectures of Hausel and Rodriguez-Villegas $[27,28]$ will be briefly reviewed. In the first two cases, the localization computations will be generalized to moduli spaces of Hitchin pairs with coefficient line bundle $L$ of degree $d(L)=2 g-2+p, p \geq 0$. As in Section 2.2, $L=K_{X}$ if $p=0$.

For $(r, e) \in \mathbb{Z}_{\geq 1} \times \mathbb{Z}$ coprime there is a smooth quasi-projective moduli space $H(X, L, r, e)$ parameterizing isomorphism classes of stable pairs $(E, \Phi), E$ is a locally free sheaf on $X$ of rank $r$ and degree $e$ and $\Phi: E \rightarrow$ $E \otimes_{X} L$ is a morphism of sheaves.

There is a torus action $\mathbb{C}^{\times} \times H(X, L, r, e) \rightarrow H(X, L, r, e), t \times(E, \Phi) \rightarrow$ $(E, t \Phi)$. The fixed points of the torus action are stable pairs of the form

$$
E \simeq \bigoplus_{i=0}^{n} E_{i}, \quad \Phi=\bigoplus_{i=0}^{n-1} \varphi_{i}
$$

where $\varphi_{i}: E_{i} \rightarrow E_{i+1} \otimes_{X} L, i=0, \ldots, n-1$, all other components being trivial. Note that the direct summand $E_{i}, 0 \leq i \leq n$, corresponds to the $\mathbb{C}^{\times}$ character $t \rightarrow t^{-i}$. If $n=0, \Phi=0$, and $E=E_{0}$ must be stable bundle on $X$.

For $(r, e) \in \mathbb{Z}_{\geq 1} \times \mathbb{Z}$ coprime the torus fixed locus is smooth. Given any connected component $\Xi$ of the fixed locus, the normal bundle to $\Xi$ is isomorphic to the moving part of the tangent bundle to $H(X, L, r, e)$ restricted to $\Xi$,

$$
N_{\Xi} \simeq T_{H(X, L, r, e)}^{m} \mid \Xi \cdot
$$

Moreover, $N_{\Xi}$ decomposes in a direct sum of the form

$$
N_{\Xi} \simeq N_{\Xi}^{+} \oplus N_{\Xi}^{-}
$$


where $N_{\Xi}^{ \pm}$is the direct sum of all $\mathbb{C}^{\times}$eigensheaves with positive, respectively, negative eigenvalues. By definition, the index of the component $\Xi$ is $r_{\Xi}^{-}=$ $r\left(N_{\Xi}^{-}\right)$.

The deformation theory of a Hitchin pair $(E, \Phi)$ is determined by the hypercohomology of the two term complex on $X$

$$
0 \rightarrow \operatorname{Hom}_{X}(E, E) \stackrel{d}{\longrightarrow} \operatorname{Hom}_{X}\left(E, E \otimes_{X} L\right) \rightarrow 0
$$

where $d(f)=\Phi \circ f-f \otimes 1_{L} \circ \Phi$. If $(E, \Phi)$ is fixed by the torus action, the equivariant version of (A.2) is

$$
0 \rightarrow \operatorname{Hom}_{X}(E, E) \stackrel{d}{\longrightarrow} Q \otimes \operatorname{Hom}_{X}\left(E, E \otimes_{X} L\right) \rightarrow 0
$$

where $E$ is of the form (A.1) and $Q$ is the irreducible representation of $\mathbb{C}^{\times}$ with character $t \rightarrow t$. If $(E, \Phi)$ is a stable pair with $(r, e)$ coprime, the 0 th hypercohomology group of (A.1) is isomorphic to $\mathbb{C}$ while the 2 nd hypercohomology group vanishes. The 1st hypercohomology group is isomorphic to the tangent space $T_{[(E, \Phi)]} H(X, L, r, e)$.

The localization computations of the Hodge polynomial of the moduli space of stable pairs of types $(2,1)$ and $(3,1)$ are reviewed below.

\section{A.1. Rank $r=2$}

Let $e=1$. Then a fixed pair is either of the form $(E, 0)$, with $E$ a stable bundle of type $(2,1)$ on $X$ or

$$
E=E_{0} \oplus E_{1}, \quad \Phi=\left[\begin{array}{cc}
0 & 0 \\
\varphi_{0} & 0
\end{array}\right]
$$

with $E_{0}, E_{1}$ line bundles of degrees $e_{0}, e_{1}, e_{0}+e_{1}=1$ and $\varphi_{0}: E_{0} \rightarrow E_{1} \otimes_{X}$ $L$ a nonzero morphism. In the second case the stability condition is equivalent to $e_{1} \leq e_{0}$, while $\varphi \neq 0$ implies $e_{0} \leq e_{1}+2 g-2+p$. Therefore

$$
\frac{1}{2} \leq e_{0} \leq g+\frac{p-1}{2}
$$

In conclusion the fixed locus is a union of the form

$$
H(X, L, 2,1)^{\mathbb{C}^{\times}} \simeq M(2,1) \cup \bigcup_{e_{0}=1}^{g+[(p-1) / 2]} J_{e_{0}}(X) \times S^{2 g-1+p-2 e_{0}}(X)
$$


where $M(2,1)$ denotes the moduli space of stable bundles of type $(2,1)$ on $X$, a smooth projective variety. An elementary computation shows that $M(2,1)$ has index 0 while each component $J_{e_{0}}(X) \times S^{2 g-1+p-2 e_{0}}(X)$ has index $2 e_{0}+g-2$, independent of $p$. Then the Hodge polynomial of the moduli space of Hitchin pairs is

$$
\begin{aligned}
& H_{(u, v)}(H(X, L, 2,1)) \\
& =H_{(u, v)}(M(2,1))+\sum_{e_{0}=1}^{g+[(p-1) / 2]} u^{2 e_{0}+g-2} v^{2 e_{0}+g-2}(1+u)^{g} \\
& \quad \times(1+v)^{g} H_{(u, v)}\left(S^{2 g-1+p-2 e_{0}}(X)\right) .
\end{aligned}
$$

Moreover, according to $[9,18,48]$, the Hodge polynomial of $M(2,1)$ is

(A.5) $\quad H_{(u, v)}(M(2,1))=(1+u)^{g}(1+v)^{g} \frac{(1+u)^{g}(1+v)^{g}}{(1-u v)\left(1-u^{2} v^{2}\right)}$

while the generating function for the Hodge polynomial of symmetric products is

$$
\sum_{n=0}^{\infty} x^{n} H_{(u, v)}\left(S^{n}(X)\right)=\frac{(1+x u)^{g}(1+x v)^{g}}{(1-x)(1-x u v)}
$$

Repeating the computations of [29] in the present context yields

$$
\begin{aligned}
& H_{(u, v)}(H(X, L, 2,1)) \\
& =(1+u)^{g}(1+v)^{g}\left[\frac{\left(1+u^{2} v\right)^{g}\left(1+u v^{2}\right)^{g}}{(1-u v)\left(1-u^{2} v^{2}\right)}+\frac{(-1)^{p+1}}{4}(u v)^{2 g-2+p}\right. \\
& \quad \times \frac{(1-u)^{g}(1-v)^{g}}{1+u v}+(u v)^{2 g-2+p} \frac{(1+u)^{g}(1+v)^{g}}{2(1-u v)} \\
& \left.\quad \times\left(\frac{g}{1+u}+\frac{g}{1+v}-\frac{1}{1-u v}-(2 g-2+p)-\frac{1}{2}\right)\right] .
\end{aligned}
$$

\section{A.2. Rank $r=3$}

In this case the computation of the Poincaré polynomial has been done in [24]. Let $e=1$ for concreteness; $e=2$ is analogous. The classification of fixed loci is more involved. There are four types of components. 
(I) $(E, \Phi)=(E, 0)$ with $E$ a rank 3 bundle on $X$ of degree $e=1$. This component is isomorphic to the moduli space $M(3,1)$ of stable rank 3 bundles on $X$ of degree $e=1$, which is a smooth projective variety. Moreover, it has index 0 and according to $[18,46]$

(A.8)

$$
\begin{aligned}
H_{(u, v)}(M(3,1))= & \frac{(1+u)^{g}(1+v)^{g}}{(1-u v)\left(1-u^{2} v^{2}\right)^{2}\left(1-u^{3} v^{3}\right)} \\
& \times\left[\left(1+u^{2} v^{3}\right)^{g}\left(1+u^{3} v^{2}\right)^{g}\left(1+u v^{2}\right)^{g}\left(1+u^{2} v\right)^{g}\right. \\
& -(u v)^{2 g-1}(1+u v)^{2}(1+u)^{g}(1+v)^{g}\left(1+u v^{2}\right)^{g}\left(1+u^{2} v\right)^{g} \\
& \left.+(u v)^{3 g-1}\left(1+u v+u^{2} v^{2}\right)(1+u)^{2 g}(1+v)^{2 g}\right]
\end{aligned}
$$

(II) $E=E_{0} \oplus E_{1}, \Phi=\left[\begin{array}{cc}0 & 0 \\ \varphi_{0} & 0\end{array}\right], E_{0}$ a degree $e_{0}$ line bundle, $E_{1}$ a bundle of type $\left(2, e_{1}\right)$ on $X$, and $\varphi_{0}: E_{0} \rightarrow E_{1} \otimes_{X} L$ a nontrivial morphism. Obviously $e_{0}+e_{1}=e$. The stability condition is equivalent to the following two conditions:

- $e / 3 \leq e_{0} \leq e / 3+g-1+p / 2$, which for $e=1$ yields $1 \leq e_{0} \leq g+$ $p / 2-2 / 3$.

- The data $\left(E_{1} \otimes L \otimes E_{0}^{-1}, \varphi_{0} \otimes 1_{E_{0}^{-1}}\right)$ is a $\sigma$-stable Thaddeus pair of type $\left(2, e-3 e_{0}+2(2 g-2+p)\right)=\left(2,1-3 e_{0}+2(2 g-2+p)\right) \quad$ (no fixed determinant) where $\sigma=e_{0} / 2-e / 6=e_{0} / 2-1 / 6$.

The index equals $3 e_{0}-e+2 g-2=3 e_{0}+2 g-3$, independent of $p$. Therefore, repeating the computation in [60, Section 4], it follows that the contribution of fixed loci of this type to the Hodge polynomial is

$$
\begin{aligned}
& H_{(u, v)}\left(\Xi^{\mathrm{II}}\left(e_{0}\right)\right)=(u v)^{3 e_{0}+2 g-3} \frac{(1+u)^{2 g}(1+v)^{2 g}}{1-u v} \\
& \operatorname{Coeff}_{x^{i}}\left[\left(\frac{(u v)^{e_{0}+g}}{x u^{2} v^{2}-1}-\frac{(u v)^{2 g-1-2 e_{0}+p}}{x-u v}\right) \frac{(1+x u)^{g}(1+x v)^{g}}{(1-x)(1-x u v)}\right]
\end{aligned}
$$

where $i=-2 e_{0}+2 g-2+p$.

(III) $E=E_{0} \oplus E_{1}, \Phi=\left[\begin{array}{cc}0 & 0 \\ \varphi_{0} & 0\end{array}\right], E_{0}$ a bundle of type $\left(2, e_{0}\right)$ and $E_{1}$ a degree $e_{1}$ line bundle on $X, e_{0}+e_{1}=1$. In this case the dual pair $\left(E^{\vee}, \Phi^{\vee} \otimes\right.$ $\left.1_{L}\right)$ is a stable fixed pair of type (II) with numerical invariants $(3,-e)=$ $(3,-1)$. Such fixed loci are labeled by an integer $\bar{e}_{0}, 0 \leq \bar{e}_{0} \leq g+p / 2-4 / 3$ and their index is $3 \bar{e}_{0}+2 g-1$. Therefore their contribution to the Hodge 
polynomial is

$$
\begin{aligned}
& H_{(u, v)}\left(\Xi^{\mathrm{III}}\left(\bar{e}_{0}\right)\right)=(u v)^{3 \bar{e}_{0}+2 g-1} \frac{(1+u)^{2 g}(1+v)^{2 g}}{1-u v} \\
& \operatorname{Coeff}_{x^{i}}\left[\left(\frac{(u v)^{\bar{e}_{0}+g}}{x u^{2} v^{2}-1}-\frac{(u v)^{2 g-2-2 e_{0}+p}}{x-u v}\right) \frac{(1+x u)^{g}(1+x v)^{g}}{(1-x)(1-x u v)}\right]
\end{aligned}
$$

where $i=-2 \bar{e}_{0}+2 g-3+p$.

(IV) $E=E_{0} \oplus E_{1} \oplus E_{2}, \Phi=\left[\begin{array}{ccc}0 & 0 & 0 \\ \varphi_{0} & 0 & 0 \\ 0 & \varphi_{1} & 0\end{array}\right], E_{0}, E_{1}, E_{2}$ line bundles of degrees $e_{0}, e_{1}, e_{2}$ on $X, e_{0}+e_{1}+e_{2}=e=1$, and $\varphi_{0}: E_{0} \rightarrow E_{1} \otimes_{X} L, \varphi_{1}$ : $E_{1} \rightarrow E_{2} \otimes_{X} L$ nontrivial morphisms. Let $m_{1}=e_{1}-e_{0}+2 g-2+p, m_{2}=$ $e_{2}-e_{0}+2 g 2+p$. Then the stability conditions are equivalent to

$m_{1}, m_{2} \geq 0, \quad m_{1}+2 m_{2} \leq 3(2 g-2+p), \quad 2 m_{1}+m_{2} \leq 3(2 g-2+p)$.

In addition, the following constraint holds by construction:

$$
m_{1}+2 m_{2} \equiv-e(\bmod 3)
$$

Fixed loci of this type are isomorphic to a direct product of the form $J_{e_{0}}(X) \times S^{m_{1}}(X) \times S^{m_{2}}(X)$. The index is $8 g-8+3 p-m_{1}-m_{2}$. Therefore their contribution to the Hodge polynomial is

$$
\begin{aligned}
& H_{(u, v)}\left(\Xi_{\left(m_{1}, m_{2}\right)}^{\mathrm{IV}}\right)=(u v)^{8 g-8+3 p-m_{1}-m_{2}}(1+u)^{g}(1+v)^{g} \\
& \operatorname{Coeff}_{x^{m_{1}}}\left(\frac{(1+x u)^{g}(1+x v)^{g}}{(1-x)(1-x u v)}\right) \operatorname{Coeff}_{x^{m_{2}}}\left(\frac{(1+x u)^{g}(1+x v)^{g}}{(1-x)(1-x u v)}\right) .
\end{aligned}
$$

In conclusion

$$
\begin{gathered}
H_{(u, v)}(H(X, L, 3,1))=H_{(u, v)}(M(3,1))+\sum_{e_{0}=1}^{g+[p / 2-2 / 3]} H_{(u, v)}\left(\Xi^{\mathrm{II}}\left(e_{0}\right)\right) \\
+\sum_{\bar{e}_{0}=0}^{g+[p / 2-4 / 3]} H_{(u, v)}\left(\Xi^{\mathrm{III}}\left(\bar{e}_{0}\right)\right)+\sum_{\substack{m_{1}, m_{2} \geq 0 \\
2 m_{1}+m_{2} \leq 6 g-6+3 p \\
m_{1}+2 m_{2} \leq 6 g-6+3 p \\
m_{1}+2 m_{2} \equiv 2(3)}} H_{(u, v)}\left(\Xi_{\left(m_{1}, m_{2}\right)}^{\mathrm{IV}}\right) .
\end{gathered}
$$




\section{A.3. Hausel-Rodriguez-Villegas formula}

This subsection is a brief summary of the formulas of Hausel and RodriguezVillegas [27, 28] for the Poincaré, respectively, Hodge polynomial of the moduli space $H\left(X, K_{X}, r, e\right)$ with $(r, e) \in \mathbb{Z}_{\geq} \times \mathbb{Z}$ coprime. Construct the following formal series:

$$
\mathcal{Z}(q, x, y, T)=1+\sum_{k \geq 1} T^{k} A_{k}(q, x, y)=1+\sum_{k \geq 1} T^{k}\left(\sum_{|Y|=k} A_{Y}(q, x, y)\right)
$$

where

$$
A_{Y}(q, x, y)=\prod_{z \in Y} \frac{(q x y)^{l(z)(2-2 g)}\left(1+q^{h(z)} y^{l(z)} x^{l(z)+1}\right)^{g}\left(1+q^{h(z)} x^{l(z)} y^{l(z)+1}\right)^{g}}{\left(1-q^{h(z)}(x y)^{l(z)+1}\right)\left(1-q^{h(z)}(x y)^{l(z)}\right)} .
$$

where for $z=(i, j) \in Y$ :

$$
a(z)=Y_{i}-j, l(z)=Y_{j}^{t}-i, h(z)=a(z)+l(z)+1 .
$$

Define $H_{r}(q, x, y)$ in terms of the following recursive formula:

$$
\begin{aligned}
& \sum_{r \geq 1} \sum_{k \geq 1} H_{r}\left(q^{k},-(-x)^{k},-(-y)^{k}\right) B_{r}\left(q^{k},-(-x)^{k},-(-y)^{k}\right) \frac{T^{k r}}{k} \\
& \quad=\log \mathcal{Z}(q, x, y, T)
\end{aligned}
$$

by comparing the coefficient of $T^{n k}$, where:

$$
B_{r}(q, x, y)=\frac{(q x y)^{(1-g) r(r-1)}(1+q x)^{g}(1+q y)^{g}}{(1-q x y)(1-q)}
$$

Then

$$
E_{r}(u, v)=H_{r}(1, u, v)
$$

is conjectured in [27] to be Hodge polynomial of the moduli space $H\left(X, K_{X}, r, e\right)$.

\section{Appendix B. Recursion results for Hodge polynomials}

This section is basically a list of results for the Hodge polynomials of the moduli spaces of Hitchin pairs determined by the double refinement the 
recursion formula (1.8) and Conjecture (1.6). According to Conjecture (1.6) the building blocks of asymptotic refined ADHM invariants are

$$
\begin{aligned}
\Omega_{\square}^{(p)}= & (-1)^{p}(u v)^{(1-g) / 2} \frac{\left(1-\lambda\left(u v^{-1}\right)^{1 / 2}\right)^{g}\left(1-\lambda\left(u^{-1} v\right)^{1 / 2}\right)^{g}}{\left(1-\lambda(u v)^{1 / 2}\right)\left(1-\lambda(u v)^{-1 / 2}\right)} \\
\Omega_{\square}^{(p)}(\lambda, u, v)= & (u v)^{1-g-p / 2} \lambda^{-p+2-2 g} G\left(\lambda^{2}(u v)^{1 / 2},(u v)^{1 / 2},\left(u v^{-1}\right)^{1 / 2}\right) \\
& \times G\left(\lambda,(u v)^{1 / 2},\left(u v^{-1}\right)^{1 / 2}\right) \\
\Omega_{\boxminus}^{(p)}(\lambda, u, v)= & (u v)^{-p / 2} \lambda^{p} G\left(\lambda^{2}(u v)^{-1 / 2},(u v)^{1 / 2},\left(u v^{-1}\right)^{1 / 2}\right) \\
& \times G\left(\lambda,(u v)^{1 / 2},\left(u v^{-1}\right)^{1 / 2}\right) \\
\Omega_{\square}^{(p)}(\lambda, u, v)= & (-1)^{p}\left(\lambda(u v)^{1 / 2}\right)^{-3 p+6(1-g)} G\left(\lambda^{3} u v,(u v)^{1 / 2},\left(u v^{-1}\right)^{1 / 2}\right) \\
& \times G\left(\lambda^{2}(u v)^{1 / 2},(u v)^{1 / 2},\left(u v^{-1}\right)^{1 / 2}\right) G\left(\lambda,(u v)^{1 / 2},\left(u v^{-1}\right)^{1 / 2}\right) \\
\Omega_{\boxminus}^{(p)}(\lambda, u, v)= & (-1)^{p}\left(\lambda^{-1}(u v)^{1 / 2}\right)^{-3 p} G\left(\lambda^{3}(u v)^{-1},(u v)^{1 / 2},\left(u v^{-1}\right)^{1 / 2}\right) \\
& \times G\left(\lambda^{2}(u v)^{-1 / 2},(u v)^{1 / 2},\left(u v^{-1}\right)^{1 / 2}\right) G\left(\lambda,(u v)^{1 / 2},\left(u v^{-1}\right)^{1 / 2}\right) \\
\Omega_{\boxminus}^{(p)}(\lambda, u, v)= & (-1)^{p}(u v)^{-p}\left(\lambda(u v)^{1 / 2}\right)^{2-2 g} G\left(\lambda^{3},(u v)^{1 / 2},\left(u v^{-1}\right)^{1 / 2}\right) \\
& \times G\left(\lambda,(u v)^{1 / 2},\left(u v^{-1}\right)^{1 / 2}\right)^{2}
\end{aligned}
$$

where

$$
G(q, z, w)=z^{(1-g)} \frac{(1-q w)^{g}\left(1-q w^{-1}\right)^{g}}{(1-q z)\left(1-q z^{-1}\right)} .
$$

(1) $g=2$

$$
\begin{aligned}
\widetilde{H}^{(0)}(2,1)(u, v)= & \frac{(1-u)^{2}(1-v)^{2}}{(\sqrt{u v})^{9}}(1+u v)\left(1-2 u^{2} v-2 u v^{2}+2 u^{2} v^{2}\right) \\
\widetilde{H}^{(0)}(2,0)(u, v)= & \frac{(1-u)^{2}(1-v)^{2}}{2(\sqrt{u v})^{9}(1+u v)}\left(2+4 u v-4 u^{2} v\right. \\
& -4 u v^{2}+7 u^{2} v^{2}-6 u^{3} v^{2}+u^{4} v^{2}-6 u^{2} v^{3}+12 u^{3} v^{3} \\
& \left.-2 u^{4} v^{3}+u^{2} v^{4}-2 u^{3} v^{4}+5 u^{4} v^{4}\right) \\
\widetilde{H}^{(0)}(3,1)(u, v)= & \frac{(1-u)^{2}(1-v)^{2}}{(\sqrt{u v})^{19}}\left(1+u v-2 u^{2} v\right. \\
& -2 u v^{2}+3 u^{2} v^{2}-4 u^{3} v^{2}+u^{4} v^{2}-4 u^{2} v^{3}+8 u^{3} v^{3} \\
& -8 u^{4} v^{3}+5 u^{5} v^{3}+u^{2} v^{4}-8 u^{3} v^{4}+19 u^{4} v^{4}-16 u^{5} v^{4} \\
& +8 u^{6} v^{4}-2 u^{7} v^{4}+5 u^{3} v^{5}-16 u^{4} v^{5}+32 u^{5} v^{5}-30 u^{6} v^{5} \\
& +12 u^{7} v^{5}-2 u^{8} v^{5}+8 u^{4} v^{6}-30 u^{5} v^{6}+43 u^{6} v^{6}-32 u^{7} v^{6}
\end{aligned}
$$




$$
\begin{aligned}
& +8 u^{8} v^{6}-2 u^{4} v^{7}+12 u^{5} v^{7}-32 u^{6} v^{7}+32 u^{7} v^{7}-12 u^{8} v^{7} \\
& \left.-2 u^{5} v^{8}+8 u^{6} v^{8}-12 u^{7} v^{8}+6 u^{8} v^{8}\right) \\
& \widetilde{H}^{(1)}(2,1)(u, v)=\frac{(1-u)^{2}(1-v)^{2}}{(\sqrt{u v})^{11}}\left(1+u v-2 u^{2} v-2 u v^{2}\right. \\
& +2 u^{2} v^{2}-2 u^{3} v^{2}+u^{4} v^{2}-2 u^{2} v^{3}+6 u^{3} v^{3} \\
& \left.-2 u^{4} v^{3}+u^{2} v^{4}-2 u^{3} v^{4}+2 u^{4} v^{4}\right) \\
& \widetilde{H}^{(1)}(2,0)(u, v)=\frac{(1-u)^{2}(1-v)^{2}}{2(\sqrt{u v})^{11}(1+u v)}\left(2+4 u v-4 u^{2} v-4 u v^{2}\right. \\
& +6 u^{2} v^{2}-8 u^{3} v^{2}+2 u^{4} v^{2}-8 u^{2} v^{3} \\
& +15 u^{3} v^{3}-10 u^{4} v^{3}+u^{5} v^{3}+2 u^{2} v^{4}-10 u^{3} v^{4}+12 u^{4} v^{4} \\
& \left.-6 u^{5} v^{4}+u^{3} v^{5}-6 u^{4} v^{5}+3 u^{5} v^{5}\right) \\
& \widetilde{H}^{(1)}(3,1)(u, v)=\frac{(1-u)^{2}(1-v)^{2}}{(\sqrt{u v})^{25}}\left(6 v^{11} u^{11}-18 v^{10} u^{11}+18 v^{9} u^{11}-8 v^{8} u^{11}\right. \\
& +v^{7} u^{11}-18 v^{11} u^{10}+60 v^{10} u^{10}-76 v^{9} u^{10}+42 v^{8} u^{10} \\
& -10 v^{7} u^{10}+v^{6} u^{10}+18 v^{11} u^{9}-76 v^{10} u^{9}+121 v^{9} u^{9} \\
& -98 v^{8} u^{9}+42 v^{7} u^{9}-8 v^{6} u^{9}-8 v^{11} u^{8}+42 v^{10} u^{8} \\
& -98 v^{9} u^{8}+124 v^{8} u^{8}-84 v^{7} u^{8}+29 v^{6} u^{8}-4 v^{5} u^{8}+v^{11} u^{7} \\
& -10 v^{10} u^{7}+42 v^{9} u^{7}-84 v^{8} u^{7}+91 v^{7} u^{7}-56 v^{6} u^{7}+17 v^{5} u^{7} \\
& -2 v^{4} u^{7}+v^{10} u^{6}-8 v^{9} u^{6}+29 v^{8} u^{6}-56 v^{7} u^{6}+58 v^{6} u^{6} \\
& -34 v^{5} u^{6}+8 v^{4} u^{6}-4 v^{8} u^{5}+17 v^{7} u^{5}-34 v^{6} u^{5}+33 v^{5} u^{5} \\
& -16 v^{4} u^{5}+5 v^{3} u^{5}-2 v^{7} u^{4}+8 v^{6} u^{4}-16 v^{5} u^{4}+19 v^{4} u^{4} \\
& -8 v^{3} u^{4}+v^{2} u^{4}+5 v^{5} u^{3}-8 v^{4} u^{3}+8 v^{3} u^{3}-4 v^{2} u^{3}+v^{4} u^{2} \\
& \left.-4 v^{3} u^{2}+3 v^{2} u^{2}-2 v u^{2}-2 v^{2} u+v u+1\right) \\
& \widetilde{H}^{(2)}(2,1)(u, v)=\frac{(1-u)^{2}(1-v)^{2}}{(\sqrt{u v})^{13}}(1+u v)\left(1-2 u^{2} v-2 u v^{2}+2 u^{2} v^{2}+u^{4} v^{2}\right. \\
& \left.+4 u^{3} v^{3}-4 u^{4} v^{3}+u^{2} v^{4}-4 u^{3} v^{4}+2 u^{4} v^{4}\right) \\
& \widetilde{H}^{(2)}(2,0)(u, v)=\frac{(1-u)^{2}(1-v)^{2}}{2(\sqrt{u v})^{13}(1+u v)}\left(2+4 u v-4 u^{2} v-4 u v^{2}+6 u^{2} v^{2}\right. \\
& -8 u^{3} v^{2}+2 u^{4} v^{2}-8 u^{2} v^{3}+16 u^{3} v^{3}-12 u^{4} v^{3}+4 u^{5} v^{3} \\
& +2 u^{2} v^{4}-12 u^{3} v^{4}+25 u^{4} v^{4}-14 u^{5} v^{4}+3 u^{6} v^{4}+4 u^{3} v^{5} \\
& \left.-14 u^{4} v^{5}+20 u^{5} v^{5}-6 u^{6} v^{5}+3 u^{4} v^{6}-6 u^{5} v^{6}+5 u^{6} v^{6}\right) \\
& \widetilde{H}^{(2)}(3,1)(u, v)=\frac{(1-u)^{2}(1-v)^{2}}{(\sqrt{u v})^{31}}\left(10 v^{14} u^{14}-32 v^{13} u^{14}+36 v^{12} u^{14}\right. \\
& -18 v^{11} u^{14}+3 v^{10} u^{14}-32 v^{14} u^{13}+112 v^{13} u^{13}
\end{aligned}
$$


Wallcrossing and cohomology of the hitchin pairs

$$
\begin{aligned}
& -154 v^{12} u^{13}+101 v^{11} u^{13}-32 v^{10} u^{13}+4 v^{9} u^{13} \\
& +36 v^{14} u^{12}-154 v^{13} u^{12}+269 v^{12} u^{12}-248 v^{11} u^{12} \\
& +123 v^{10} u^{12}-30 v^{9} u^{12}+3 v^{8} u^{12}-18 v^{14} u^{11} \\
& +101 v^{13} u^{11}-248 v^{12} u^{11}+329 v^{11} u^{11}-250 v^{10} u^{11} \\
& +109 v^{9} u^{11}-24 v^{8} u^{11}+v^{7} u^{11}+3 v^{14} u^{10}-32 v^{13} u^{10} \\
& +123 v^{12} u^{10}-250 v^{11} u^{10}+298 v^{10} u^{10}-208 v^{9} u^{10} \\
& +79 v^{8} u^{10}-14 v^{7} u^{10}+v^{6} u^{10}+4 v^{13} u^{9}-30 v^{12} u^{9} \\
& +109 v^{11} u^{9}-208 v^{10} u^{9}+226 v^{9} u^{9}-146 v^{8} u^{9} \\
& +53 v^{7} u^{9}-8 v^{6} u^{9}+3 v^{12} u^{8}-24 v^{11} u^{8}+79 v^{10} u^{8} \\
& -146 v^{9} u^{8}+159 v^{8} u^{8}-96 v^{7} u^{8}+30 v^{6} u^{8}-4 v^{5} u^{8} \\
& +v^{11} u^{7}-14 v^{10} u^{7}+53 v^{9} u^{7}-96 v^{8} u^{7}+98 v^{7} u^{7} \\
& -58 v^{6} u^{7}+17 v^{5} u^{7}-2 v^{4} u^{7}+v^{10} u^{6}-8 v^{9} u^{6} \\
& +30 v^{8} u^{6}-58 v^{7} u^{6}+59 v^{6} u^{6}-34 v^{5} u^{6}+8 v^{4} u^{6} \\
& -4 v^{8} u^{5}+17 v^{7} u^{5}-34 v^{6} u^{5}+33 v^{5} u^{5}-16 v^{4} u^{5} \\
& +5 v^{3} u^{5}-2 v^{7} u^{4}+8 v^{6} u^{4}-16 v^{5} u^{4}+19 v^{4} u^{4} \\
& -8 v^{3} u^{4}+v^{2} u^{4}+5 v^{5} u^{3}-8 v^{4} u^{3}+8 v^{3} u^{3} \\
& -4 v^{2} u^{3}+v^{4} u^{2}-4 v^{3} u^{2}+3 v^{2} u^{2}-2 v u^{2} \\
& \left.-2 v^{2} u+v u+1\right)
\end{aligned}
$$

(2) $g=3$

$$
\begin{aligned}
\widetilde{H}^{(0)}(2,1)(u, v)= & \frac{(1-u)^{3}(1-v)^{3}}{(\sqrt{u v})^{17}}\left(1+u v-3 u^{2} v-3 u v^{2}+2 u^{2} v^{2}\right. \\
& -3 u^{3} v^{2}+3 u^{4} v^{2}-3 u^{2} v^{3}+11 u^{3} v^{3}-6 u^{4} v^{3}+3 u^{5} v^{3} \\
& -u^{6} v^{3}+3 u^{2} v^{4}-6 u^{3} v^{4}+12 u^{4} v^{4}-15 u^{5} v^{4}+3 u^{6} v^{4} \\
& +3 u^{3} v^{5}-15 u^{4} v^{5}+12 u^{5} v^{5}-6 u^{6} v^{5}-u^{3} v^{6}+3 u^{4} v^{6} \\
& \left.-6 u^{5} v^{6}+3 u^{6} v^{6}\right) \\
& (1-u)^{3}(1-v)^{3} \\
\widetilde{H}^{(0)}(2,0)(u, v)= & 2(\sqrt{u v})^{17}(1+u v) \\
& -12 u^{3} v^{2}+6 u^{4} v^{2}-12 u^{2} v^{3}+26 u^{3} v^{3}-18 u^{4} v^{3} \\
& +12 u^{5} v^{3}-2 u^{6} v^{3}+6 u^{2} v^{4}-18 u^{3} v^{4}+47 u^{4} v^{4} \\
& -39 u^{5} v^{4}+15 u^{6} v^{4}-u^{7} v^{4}+12 u^{3} v^{5}-39 u^{4} v^{5} \\
& +57 u^{5} v^{5}-33 u^{6} v^{5}+9 u^{7} v^{5}-2 u^{3} v^{6}+15 u^{4} v^{6}
\end{aligned}
$$




$$
\begin{aligned}
& -33 u^{5} v^{6}+39 u^{6} v^{6}-9 u^{7} v^{6}-u^{4} v^{7} \\
& \left.+9 u^{5} v^{7}-9 u^{6} v^{7}+7 u^{7} v^{7}\right) \\
& \widetilde{H}^{(0)}(3,1)(u, v)=\frac{(1-u)^{3}(1-v)^{3}}{(\sqrt{u v})^{37}}\left(15 v^{16} u^{16}-60 v^{15} u^{16}+102 v^{14} u^{16}\right. \\
& -93 v^{13} u^{16}+45 v^{12} u^{16}-12 v^{11} u^{16}+v^{10} u^{16}-60 v^{16} u^{15} \\
& +276 v^{15} u^{15}-537 v^{14} u^{15}+549 v^{13} u^{15}-324 v^{12} u^{15} \\
& +102 v^{11} u^{15}-15 v^{10} u^{15}+v^{9} u^{15}+102 v^{16} u^{14} \\
& -537 v^{15} u^{14}+1167 v^{14} u^{14}-1407 v^{13} u^{14}+990 v^{12} u^{14} \\
& -417 v^{11} u^{14}+102 v^{10} u^{14}-12 v^{9} u^{14}-93 v^{16} u^{13} \\
& +549 v^{15} u^{13}-1407 v^{14} u^{13}+2003 v^{13} u^{13}-1776 v^{12} u^{13} \\
& +1020 v^{11} u^{13}-362 v^{10} u^{13}+72 v^{9} u^{13}-6 v^{8} u^{13} \\
& +45 v^{16} u^{12}-324 v^{15} u^{12}+990 v^{14} u^{12}-1776 v^{13} u^{12} \\
& +2069 v^{12} u^{12}-1587 v^{11} u^{12}+798 v^{10} u^{12}-251 v^{9} u^{12} \\
& +42 v^{8} u^{12}-3 v^{7} u^{12}-12 v^{16} u^{11}+102 v^{15} u^{11} \\
& -417 v^{14} u^{11}+1020 v^{13} u^{11}-1587 v^{12} u^{11}+1659 v^{11} u^{11} \\
& -1173 v^{10} u^{11}+537 v^{9} u^{11}-156 v^{8} u^{11}+21 v^{7} u^{11} \\
& +v^{16} u^{10}-15 v^{15} u^{10}+102 v^{14} u^{10}-362 v^{13} u^{10} \\
& +798 v^{12} u^{10}-1173 v^{11} u^{10}+1151 v^{10} u^{10}-777 v^{9} u^{10} \\
& +330 v^{8} u^{10}-80 v^{7} u^{10}+12 v^{6} u^{10}+v^{15} u^{9}-12 v^{14} u^{9} \\
& +72 v^{13} u^{9}-251 v^{12} u^{9}+537 v^{11} u^{9}-777 v^{10} u^{9} \\
& +731 v^{9} u^{9}-456 v^{8} u^{9}+195 v^{7} u^{9}-41 v^{6} u^{9}+3 v^{5} u^{9} \\
& -6 v^{13} u^{8}+42 v^{12} u^{8}-156 v^{11} u^{8}+330 v^{10} u^{8} \\
& -456 v^{9} u^{8}+447 v^{8} u^{8}-261 v^{7} u^{8}+99 v^{6} u^{8}-21 v^{5} u^{8} \\
& -3 v^{12} u^{7}+21 v^{11} u^{7}-80 v^{10} u^{7}+195 v^{9} u^{7}-261 v^{8} u^{7} \\
& +242 v^{7} u^{7}-147 v^{6} u^{7}+45 v^{5} u^{7}-10 v^{4} u^{7}+12 v^{10} u^{6} \\
& -41 v^{9} u^{6}+99 v^{8} u^{6}-147 v^{7} u^{6}+122 v^{6} u^{6}-78 v^{5} u^{6} \\
& +21 v^{4} u^{6}-v^{3} u^{6}+3 v^{9} u^{5}-21 v^{8} u^{5}+45 v^{7} u^{5} \\
& -78 v^{6} u^{5}+63 v^{5} u^{5}-30 v^{4} u^{5}+12 v^{3} u^{5}-10 v^{7} u^{4} \\
& +21 v^{6} u^{4}-30 v^{5} u^{4}+34 v^{4} u^{4}-12 v^{3} u^{4} \\
& +3 v^{2} u^{4}-v^{6} u^{3}+12 v^{5} u^{3}-12 v^{4} u^{3}+13 v^{3} u^{3} \\
& -6 v^{2} u^{3}+3 v^{4} u^{2}-6 v^{3} u^{2}+3 v^{2} u^{2} \\
& \left.-3 v u^{2}-3 v^{2} u+v u+1\right)
\end{aligned}
$$




$$
\begin{aligned}
& \widetilde{H}^{(1)}(2,1)(u, v)=\frac{(1-u)^{3}(1-v)^{3}}{(\sqrt{u v})^{19}}(1+u v)\left(1-3 u^{2} v-3 u v^{2}\right. \\
& +2 u^{2} v^{2}+3 u^{4} v^{2}+9 u^{3} v^{3}-6 u^{4} v^{3} \\
& -u^{6} v^{3}+3 u^{2} v^{4}-6 u^{3} v^{4}+3 u^{4} v^{4}-9 u^{5} v^{4} \\
& +6 u^{6} v^{4}-9 u^{4} v^{5}+18 u^{5} v^{5} \\
& \left.-6 u^{6} v^{5}-u^{3} v^{6}+6 u^{4} v^{6}-6 u^{5} v^{6}+3 u^{6} v^{6}\right) \\
& \widetilde{H}^{(1)}(2,0)(u, v)=\frac{(1-u)^{3}(1-v)^{3}}{2(\sqrt{u v})^{19}(1+u v)}\left(2+4 u v-6 u^{2} v-6 u v^{2}\right. \\
& +6 u^{2} v^{2}-12 u^{3} v^{2}+6 u^{4} v^{2}-12 u^{2} v^{3} \\
& +26 u^{3} v^{3}-18 u^{4} v^{3}+12 u^{5} v^{3}-2 u^{6} v^{3}+6 u^{2} v^{4} \\
& -18 u^{3} v^{4}+46 u^{4} v^{4}-42 u^{5} v^{4}+18 u^{6} v^{4}-4 u^{7} v^{4} \\
& +12 u^{3} v^{5}-42 u^{4} v^{5}+65 u^{5} v^{5}-63 u^{6} v^{5}+21 u^{7} v^{5} \\
& -3 u^{8} v^{5}-2 u^{3} v^{6}+18 u^{4} v^{6}-63 u^{5} v^{6}+75 u^{6} v^{6} \\
& -51 u^{7} v^{6}+9 u^{8} v^{6}-4 u^{4} v^{7}+21 u^{5} v^{7} \\
& -51 u^{6} v^{7}+39 u^{7} v^{7}-15 u^{8} v^{7}-3 u^{5} v^{8} \\
& \left.+9 u^{6} v^{8}-15 u^{7} v^{8}+5 u^{8} v^{8}\right) \\
& \widetilde{H}^{(2)}(2,1)(u, v)=\frac{(1-u)^{3}(1-v)^{3}}{(\sqrt{u v})^{21}}\left(1+u v-3 u^{2} v-3 u v^{2}\right. \\
& +2 u^{2} v^{2}-3 u^{3} v^{2}+3 u^{4} v^{2}-3 u^{2} v^{3}+11 u^{3} v^{3} \\
& -6 u^{4} v^{3}+3 u^{5} v^{3}-u^{6} v^{3}+3 u^{2} v^{4}-6 u^{3} v^{4} \\
& +12 u^{4} v^{4}-15 u^{5} v^{4}+6 u^{6} v^{4}-u^{7} v^{4}+3 u^{3} v^{5} \\
& -15 u^{4} v^{5}+21 u^{5} v^{5}-18 u^{6} v^{5}+9 u^{7} v^{5}-2 u^{8} v^{5} \\
& -u^{3} v^{6}+6 u^{4} v^{6}-18 u^{5} v^{6}+30 u^{6} v^{6}-24 u^{7} v^{6} \\
& +6 u^{8} v^{6}-u^{4} v^{7}+9 u^{5} v^{7}-24 u^{6} v^{7}+21 u^{7} v^{7} \\
& \left.-9 u^{8} v^{7}-2 u^{5} v^{8}+6 u^{6} v^{8}-9 u^{7} v^{8}+3 u^{8} v^{8}\right) \\
& \widetilde{H}^{(2)}(2,0)(u, v)=\frac{(1-u)^{3}(1-v)^{3}}{2(\sqrt{u v})^{21}(1+u v)}\left(2+4 u v-6 u^{2} v-6 u v^{2}\right. \\
& +6 u^{2} v^{2}-12 u^{3} v^{2}+6 u^{4} v^{2}-12 u^{2} v^{3}+26 u^{3} v^{3} \\
& -18 u^{4} v^{3}+12 u^{5} v^{3}-2 u^{6} v^{3}+6 u^{2} v^{4}-18 u^{3} v^{4} \\
& +46 u^{4} v^{4}-42 u^{5} v^{4}+18 u^{6} v^{4}-4 u^{7} v^{4}+12 u^{3} v^{5} \\
& -42 u^{4} v^{5}+66 u^{5} v^{5}-66 u^{6} v^{5}+30 u^{7} v^{5}-6 u^{8} v^{5}
\end{aligned}
$$




$$
\begin{aligned}
& -2 u^{3} v^{6}+18 u^{4} v^{6}-66 u^{5} v^{6}+103 u^{6} v^{6}-81 u^{7} v^{6} \\
& +33 u^{8} v^{6}-3 u^{9} v^{6}-4 u^{4} v^{7}+30 u^{5} v^{7}-81 u^{6} v^{7} \\
& +111 u^{7} v^{7}-57 u^{8} v^{7}+15 u^{9} v^{7}-6 u^{5} v^{8} \\
& +33 u^{6} v^{8}-57 u^{7} v^{8}+57 u^{8} v^{8}-15 u^{9} v^{8} \\
& \left.-3 u^{6} v^{9}+15 u^{7} v^{9}-15 u^{8} v^{9}+7 u^{9} v^{9}\right) .
\end{aligned}
$$

\section{References}

[1] O. Garcia-Prada, J. Heinloth and A. Schmitt, 'On the motives of moduli of chains and Higgs bundles', preprint, arXiv:11004.5558

[2] M. F. Atiyah and R. Bott, The Yang-Mills equations over Riemann surfaces, Philos. Trans. R. Soc. London Ser. A308(1505) (1983), 523615.

[3] K. Behrend, Donaldson-Thomas type invariants via microlocal geometry, Ann. Math. (2) 170(3) (2009), 1307-1338.

[4] K. Behrend, J. Bryan and B. Szendroi, Motivic degree zero DonaldsonThomas invariants, arXiv:0909.5088.

[5] I. Biswas and S. Ramanan, An infinitesimal study of the moduli of Hitchin pairs, J. London Math. Soc. (2) 49(2) (1994), 219-231.

[6] S. Cecotti and C. Vafa, BPS wall crossing and topological strings, hep-th/0910.2615.

[7] W.-y. Chuang, D.-E. Diaconescu and G. Pan, Chamber structure and wallcrossing in the ADHM theory of curves II, arXiv:0908.1119.

[8] W.-Y. Chuang, D.-E. Diaconescu and G. Pan, Rank two ADHM invariants and wallcrossing, Commun. Number Theory Phys. 4(2) (2010), $417-461$.

[9] S. Del Baño, On the motive of moduli spaces of rank two vector bundles over a curve, Compositio Math. 131(1) (2002), 1-30.

[10] F. Denef and G. W. Moore, Split states, entropy enigmas, holes and halos, arXi.org:hep-th/0702146.

[11] U. V. Desale and S. Ramanan, Poincaré polynomials of the variety of stable bundles, Math. Ann. 216(3) (1975), 233-244. 
[12] D.-E. Diaconescu, Chamber structure and wallcrossing in the ADHM theory of curves I, arXiv:0904.4451.

[13] D. E. Diaconescu, Moduli of ADHM sheaves and local DonaldsonThomas theory, arXiv.org:0801.0820.

[14] D.-E. Diaconescu, B. Florea and N. Saulina, A vertex formalism for local ruled surfaces, Commun. Math. Phys. 265 (2006), 201-226.

[15] D. E. Diaconescu and G. W. Moore, Crossing the wall: branes vs. bundles, arXiv.org:hep-th/0706.3193.

[16] T. Dimofte and S. Gukov, Refined, motivic, and quantum, Lett. Math. Phys. 91 (2010), 1.

[17] T. Dimofte, S. Gukov and Y. Soibelman, Quantum wall crossing in $N=2$ gauge theories. arXiv:0912.1346.

[18] R. Earl and F. Kirwan, The Hodge numbers of the moduli spaces of vector bundles over a Riemann surface, Q. J. Math. 51(4) (2000), 465-483.

[19] T. Eguchi and H. Kanno, Five-dimensional gauge theories and local mirror symmetry, Nucl. Phys. B586 (2000), 331-345.

[20] T. Eguchi and H. Kanno, Topological strings and Nekrasov's formulas, JHEP 12 (2003), 006.

[21] D. Gaiotto, G. Moore and A. Neitzke, Framed BPS states, arXiv: 1006.0146.

[22] O. García-Prada, P. B. Gothen and V. Muñoz, Betti numbers of the moduli space of rank 3 parabolic Higgs bundles, Mem. Amer. Math. Soc. 187(879) (2007), viii+80.

[23] A. Gholampour and A. Sheshmani, BPS states via Higgs type bundles, in preparation.

[24] P. B. Gothen, The Betti numbers of the moduli space of stable rank 3 Higgs bundles on a Riemann surface, Int. J. Math. 5(6) (1994), 861875.

[25] L. Göttsche, Change of polarization and Hodge numbers of moduli spaces of torsion free sheaves on surfaces, Math. Z. 223(2) (1996), $247-260$.

[26] G. Harder and M. S. Narasimhan, On the cohomology groups of moduli spaces of vector bundles on curves, Math. Ann. 212 (1974/75), 215-248. 
54 Wu-yen Chuang, Duiliu-Emanuel Diaconescu and Guang Pan

[27] T. Hausel, Mirror symmetry and Langlands duality in the non-abelian Hodge theory of a curve, in 'Geometric methods in algebra and number theory', Progr. Math. Birkhäuser Boston, 235 Boston, MA, 2005, 193-217.

[28] T. Hausel and F. Rodriguez-Villegas, Mixed Hodge polynomials of character varieties, Invent. Math. 174(3) (2008), 555-624. With an appendix by Nicholas M. Katz.

[29] N. J. Hitchin, The self-duality equations on a Riemann surface, Proc. London Math. Soc. (3) 55(1) (1987), 59-126.

[30] T. J. Hollowood, A. Iqbal and C. Vafa, Matrix models, geometric engineering and elliptic Genera, JHEP 03 (2008), 069.

[31] A. Iqbal and A.-K. Kashani-Poor, Instanton counting and ChernSimons theory, Adv. Theor. Math. Phys. 7 (2004), 457-497.

[32] A. Iqbal and A.-K. Kashani-Poor, $S U(N)$ geometries and topological string amplitudes, Adv. Theor. Math. Phys. 10 (2006), 1-32.

[33] A. Iqbal, C. Kozcaz and C. Vafa, The refined topological vertex, JHEP 10 (2009), 069.

[34] D. Joyce, Configurations in abelian categories. I. Basic properties and moduli stacks, Adv. Math. 203(1) (2006), 194-255.

[35] D. Joyce, Configurations in abelian categories. II. Ringel-Hall algebras, Adv. Math. 210(2) (2007), 635-706.

[36] D. Joyce, Configurations in abelian categories. III. Stability conditions and identities, Adv. Math. 215(1) (2007), 153-219.

[37] D. Joyce, Configurations in abelian categories. IV. Invariants and changing stability conditions, Adv. Math. 217(1) (2008), 125-204.

[38] D. Joyce and Y. Song, A theory of generalized Donaldson-Thomas invariants, arxiv.org:0810.5645.

[39] S. H. Katz, D. R. Morrison and M. Ronen Plesser, Enhanced gauge symmetry in type II string theory, Nucl. Phys. B477 (1996), $105-140$.

[40] Y. Konishi, Topological strings, instantons and asymptotic forms of Gopakumar-Vafa invariants. hep-th/0312090.

[41] M. Kontsevich and Y. Soibelman, Motivic Donaldson-Thomas invariants: summary of results, arXiv:0910:4315. 
[42] M. Kontsevich and Y. Soibelman, Stability structures, DonaldsonThomas invariants and cluster transformations, arXiv.org: 0811.2435.

[43] A. E. Lawrence and N. Nekrasov, Instanton sums and five-dimensional gauge theories, Nucl. Phys. B513 (1998), 239-265.

[44] J. Li, K. Liu, and J. Zhou, Topological string partition functions as equivariant indices, Asian J. Math. 10(1) (2006), 81-114.

[45] S. Mozgovoy, 'Solutions of the motivic ADHM recursion formula', preprint, arXiv:1104.5698

[46] V. Muñoz, Hodge polynomials of the moduli spaces of rank 3 pairs, Geom. Dedicata 136 (2008), 17-46.

[47] V. Muñoz, D. Ortega and M.-J. Vázquez-Gallo, Hodge polynomials of the moduli spaces of pairs, Int. J. Math. 18(6) (2007), 695-721.

[48] V. Muñoz, D. Ortega and M.-J. Vázquez-Gallo, Hodge polynomials of the moduli spaces of triples of rank $(2,2)$, Q. J. Math. 60(2) (2009), $235-272$.

[49] K. Nagao, Refined open non-commutative Donaldson-Thomas invariants for small crepant resolutions, arxiv.org:0907.3784.

[50] H. Nakajima, Lectures on Hilbert schemes of points on surfaces, University Lecture Series, 18, American Mathematical Society, Providence, RI, 1999.

[51] H. Nakajima and K. Yoshioka, Instanton counting on blowup. I. 4-dimensional pure gauge theory, Invent. Math. 162(2) (2005), $313-355$.

[52] H. Nakajima and K. Yoshioka, Instanton counting on blowup. II. K-theoretic partition function, Transform. Groups, 10(3-4) (2005), 489-519.

[53] N. A. Nekrasov, Seiberg-Witten prepotential from instanton counting, Adv. Theor. Math. Phys. 7 (2004), 831-864.

[54] N. Nitsure, Moduli space of semistable pairs on a curve, Proc. London Math. Soc. (3) 62(2) (1991), 275-300.

[55] R. Pandharipande and R. P. Thomas, Curve counting via stable pairs in the derived category, Invent. Math. 178(2) (2009), 407-447. 
56 Wu-yen Chuang, Duiliu-Emanuel Diaconescu and Guang Pan

[56] A. Schmitt, Projective moduli for Hitchin pairs, Int. J. Math. 9(1) (1998), 107-118.

[57] C. T. Simpson, Moduli of representations of the fundamental group of a smooth projective variety. I, Inst. Hautes Études Sci. Publ. Math. (79) (1994), 47-129.

[58] C. T. Simpson, Moduli of representations of the fundamental group of a smooth projective variety. II, Inst. Hautes Études Sci. Publ. Math. (80) (1995), 1994, 5-79.

[59] Y. Tachikawa, Five-dimensional Chern-Simons terms and Nekrasov's instanton counting, JHEP 02 (2004), 050.

[60] M. Thaddeus, Stable pairs, linear systems and the Verlinde formula, Invent. Math. 117(2) (1994), 317-353.

[61] Y. Toda, On a computation of rank two Donaldson-Thomas invariants, arxiv.org:0912.2507.

[62] K. Yoshioka, The Betti numbers of the moduli space of stable sheaves of rank 2 on a ruled surface, Math. Ann. 302(3) (1995), 519-540.

[63] K. Yoshioka, Chamber structure of polarizations and the moduli of stable sheaves on a ruled surface, Int. J. Math. 7(3) (1996), 411-431.

Department of Mathematics

NATIONAL TAIWAN UNIVERSITY

TAIPEI

TAIWAN

E-mail address: wychuang@math.ntu.edu.tw

NHETC, Rutgers UNIVERSITY

PISCATAWAY, NJ 08854-0849

USA

E-mail address: duiliu@physics.rutgers.edu

Department of Physics and Astronomy

Rutgers UNIVERSITY

136 Frelinghuysen RoAd

PISCATAWAY, NJ 08554-0819

USA

E-mail address: guangpan@physics.rutgers.edu

Received December 7, 2010 